\title{
Local projections of layer Vb-to-Va are more effective in lateral than in medial entorhinal cortex
}

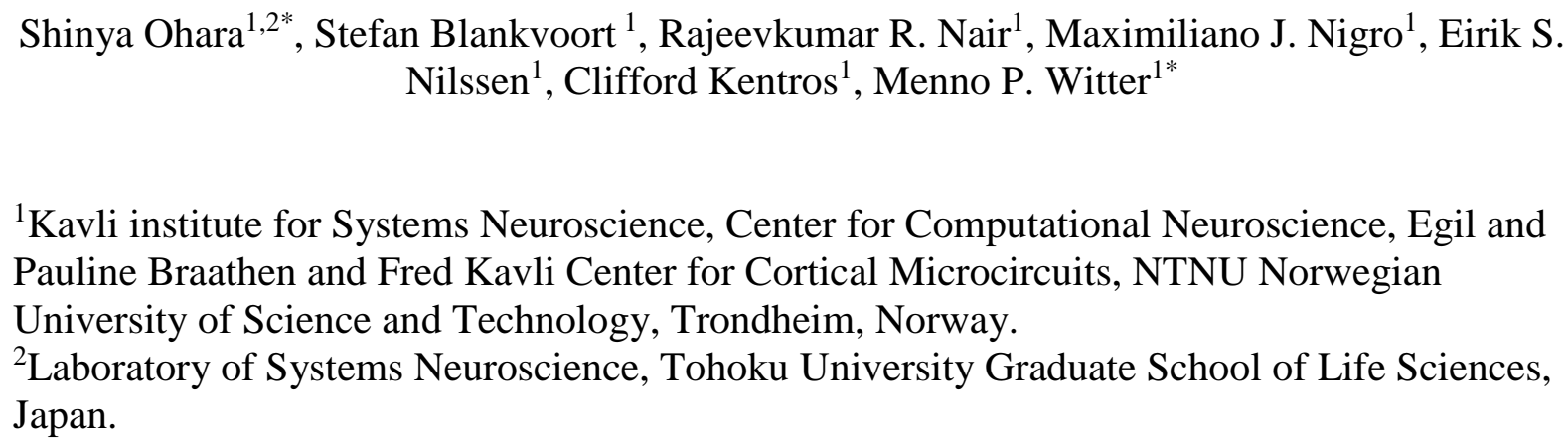

${ }^{1}$ Kavli institute for Systems Neuroscience, Center for Computational Neuroscience, Egil and Pauline Braathen and Fred Kavli Center for Cortical Microcircuits, NTNU Norwegian University of Science and Technology, Trondheim, Norway.

${ }^{2}$ Laboratory of Systems Neuroscience, Tohoku University Graduate School of Life Sciences, Japan.

* Correspondence:

\section{Menno Witter}

Kavli Institute for Systems Neuroscience

\section{Shinya Ohara}

Faculty for Medical and Health Sciences, NTNU

Postboks 8905

7491 Trondheim

Norway

Email: $\underline{\text { menno.witter@ntnu.no }}$
Laboratory of Systems Neuroscience

Tohoku University Graduate School of Life Sciences

Sendai

Japan

shinyaohara@gmail.com

Running title: Hippocampal output circuits in entorhinal cortex layer V.

Word count main text: 5519 words

Word count summary: 148 words

Number of figures: 6

Number of figure supplements: 10

Number of source files: 7

\section{Keywords}

parahippocampal region, local circuit, hippocampal-cortical output circuit, hippocampalentorhinal re-entry circuit, systems memory consolidation 


\section{Summary}

The entorhinal cortex, in particular neurons in layer $\mathrm{V}$, allegedly mediate transfer of information from the hippocampus to the neocortex, underlying long-term memory. Recently, this circuit has been shown to comprise a hippocampal output recipient layer $\mathrm{Vb}$ and a cortical projecting layer Va. With the use of in vitro electrophysiology in transgenic mice specific for layer $\mathrm{Vb}$, we assessed the presence of the thus necessary connection from layer Vb-to-Va in the functionally distinct medial (MEC) and lateral (LEC) subdivisions; MEC, particularly its dorsal part, processes allocentric spatial information, whereas the corresponding part of LEC processes information representing elements of episodes. Using identical experimental approaches, we show that connections from layer $\mathrm{Vb}$-to-Va neurons are more effective in LEC compared with dorsal MEC. This indicates that the hippocampalcortex output circuit is more effective in LEC, suggesting that episodic systems-consolidation may use LEC-derived information more than allocentric spatial information from MEC.

\section{Introduction}

Everyday memories, which include information of place, time, and content of episodes, gradually mature from an initially labile state to a more stable and long-lasting state. This memory maturation process, called memory consolidation, involves gradual reorganization of interconnected brain regions: memories that are initially depending on hippocampus become increasingly dependent on cortical networks over time (Frankland and Bontempi, 2005). Although various models have been hypothesized for this systems level consolidation, such as the standard consolidation model and multiple trace theory (Nadel and Moscovitch, 1997; Squire and Alvarez, 1995), they all share a canonical hippocampal-cortical output circuit via the entorhinal cortex (EC), which is crucial to mediate long-term memory storage and recall (Buzsáki, 1996; Eichenbaum et al., 2012). The existence of this circuit was originally proposed based on the ground-breaking report of a non-fornical hippocampal-cortical output route mediated by layer V (LV) of the EC in monkeys (Rosene and Van Hoesen, 1977), which was later confirmed also in rodents (Köhler, 1985; Kosel et al., 1982).

The EC is composed of two functionally distinct subdivisions, the lateral and medial EC (LEC and MEC respectively). MEC processes allocentric, mainly spatial information, whereas LEC represents the time and content of episodes (Deshmukh and Knierim, 2011; Hafting et al., 2005; Montchal et al., 2019; Tsao et al., 2018, 2013; Xu and Wilson, 2012). Despite these evident functional differences, both subdivisions are assumed to share the same cortical output system mediated by LV neurons. Recently we and others have shown that LV in both MEC and LEC can be genetically and connectionally divided into two sublayers: a deep layer $\mathrm{Vb}(\mathrm{LVb})$, which contains neurons receiving projections from the hippocampus, and a superficial layer $\mathrm{Va}$ (LVa), which originates the main projections out to forebrain cortical and subcortical structures (Ohara et al., 2018; Ramsden et al., 2015; Sürmeli et al., 2015; Wozny et al., 2018). These results indicate that for the hippocampal-cortical dialogue to function, we need to postulate a projection from LVb to LVa neurons. Although the existence of such a LVb-LVa circuit is supported by our previous study using transsynaptic viral tracing in rats (Ohara et al., 2018), experimental evidence for functional connectivity from LVb-to-Va in LEC and MEC is still lacking.

In the present study we examined the presence of this hypothetical intrinsic EC circuit by using a newly generated $\mathrm{LVb}$-specific transgenic (TG) mouse-line obtained with an enhancer-driven gene expression (EDGE) approach (Blankvoort et al., 2018). To compare the 
LVb intrinsic circuit between LEC and MEC, we ran identical in vitro electrophysiological and optogenetical experiments in comparable dorsal portions of LEC and MEC. To our surprise, we found differences in the postulated intrinsic LVb-LVa pathway between the two entorhinal subdivisions: the connectivity was prominent in dorsal LEC but sparse in dorsal parts of MEC. In contrast, other intrinsic circuits from LVb to layers II and III (LII and LIII), which constitute hippocampal-entorhinal re-entry circuits, are very similar in both entorhinal subdivisions. These results suggest that the canonical hippocampal-cortical output circuit that allegedly is crucial for systems consolidation is less effective or at least more complex in the allocentric navigation system. Our data point to an important deviation from the current view of how the hippocampus interacts with the neocortex and thus impacts on current theories about systems consolidation and the hippocampal memory system.

\section{Results}

\section{Characterization of $L \mathrm{Vb}$ transgenic mouse line}

Entorhinal LV can be divided into superficial LVa and deep LVb based on differences in cytoarchitectonics, connectivity and genetic markers such as purkinje cell protein 4 (PCP4) and chicken ovalbumin upstream promoter transcription factor interacting protein 2 (Ctip2) (Ohara et al., 2018; Sürmeli et al., 2015) (Figure 1—figure supplement 1, see Methods for details). To target the entorhinal LVb neurons, we used a TG mouse line (MEC-13-53D) which was obtained with the EDGE approach (Blankvoort et al., 2018). In this TG line, the tetracycline-controlled transactivator (tTA, Tet-Off) is expressed under the control of a specific enhancer and a downstream minimal promoter. To visualize the expression patterns of tTA, this line was crossed to tTA-dependent reporter mouse which express mCherry together with the tTA dependent GCaMP6.

In both LEC and MEC, mCherry-positive neurons were observed mainly in LVb $(93.2 \%$ in LEC and $82.9 \%$ in MEC) and some in layer VI (LVI; $5.1 \%$ in LEC and $16.8 \%$ in MEC) but hardly in LVa (1.7 \% in LEC and $0.3 \%$ in MEC), and none in superficial layers (Figure 1AD). The proportion of PCP4-positive LVb neurons which show tTA-driven labelling was 45.9\% in LEC and 30.9\% in MEC (Figure 1E). The tTA-driven labelling colocalized well with the PCP4-labeling (percentage of tTA-expressing neurons that were PCP4-positive was 91.7\% in LEC and $99.3 \%$ in MEC; Figure 1F), highlighting the specificity of the line. In another experiment using a GAD67 transgenic line expressing green fluorescent protein (GFP), we showed that the percentage of double-labelled (PCP4+, GAD67+) neurons among total GAD67-positive neurons is very low in both LEC and MEC (4.3\% and 2.3\% respectively, Figure 1 - figure supplement 2). This percentage of double-labelled neurons was significantly lower than in the Ctip2-stained sample in both regions (18.1\% for LEC and $7.2 \%$ for MEC). This result shows that PCP4 can be used as a marker for excitatory entorhinal LVb neurons. Occasionally PCP4-positive neurons were observed in what seems to be layer $\mathrm{Va}$, where there is a lack of continuity in the cell layer as indicated by retrograde tracing (Figure 1-figure supplement 1). Although sparse, these 'misplaced' LVb neurons were also targeted in our TG mouse line. The MEC-13-53D is thus an attractive TG mouseline to target excitatory LVb neurons in both LEC and MEC.

\section{Morphological properties of LVa/LVb neurons in LEC and MEC}

We next examined the morphological and electrophysiological properties of the LVb neurons in LEC and MEC in this TG-mouse line. Targeted LVb neurons were labelled by injecting tTA-dependent adeno-associated virus (AAV) encoding GFP (AAV2/1-TRE-Tight-GFP) into 
either LEC or MEC and filled with biocytin during whole-cell patch-clamp recordings in acute slices (Figure 1G). Consistent with our histological result showing that this line targets excitatory cells, all recorded cells showed morphological and electrophysiological properties of excitatory neurons (Figure 1, Figure 2). In line with previous studies, many MEC-LVb neurons were pyramidal cells with apical dendrites that ascended straight towards layer I (LI; Figure 1H, J) (Canto and Witter, 2012a; Hamam et al., 2000). In contrast, more than $40 \%$ of the targeted LEC-LVb neurons were tilted pyramidal neurons (Canto and Witter, 2012b; Hamam et al., 2002) with apical dendrites not extending superficially beyond LIII (Figure 1I, J). Since this latter result may result from severing of dendrites by the slicing procedure, we also examined the distribution of LVb apical dendrites in vivo. After injecting AAV2/1-TRETight-GFP in the deep layer of LEC in the TG line, the distribution of labelled dendrites of LEC-LVb neurons were examined throughout all sections (Figure 1-figure supplement 3). Even with this approach, the labelled dendrites mainly terminated in LIII and only sparsely reached layer IIb. These morphological differences indicate that MEC-LVb neurons sample inputs from different layers than LEC-LVb neurons: MEC-LVb neurons receive inputs throughout all layers, whereas LEC-LVb neurons only receive inputs innervating layer IIbVI. In contrast to LVb neurons, the morphology of LVa neurons was relatively similar in both regions: the basal dendrites extended horizontally mostly within LVa, whereas the apical dendrites reached LI (Figure 1-figure supplement 4). These morphological features of LVa neurons are in line with previous studies (Canto and Witter, 2012a, 2012b; Hamam et al., 2000, 2002; Sürmeli et al., 2015).

\section{Electrophysiological properties of LVa/LVb neurons in LEC and MEC}

Previous studies have reported that the electrophysiological profiles of LV neurons are diverse both in LEC and MEC (Canto and Witter, 2012a, 2012b; Hamam et al., 2000, 2002), but whether these different electrophysiological properties of entorhinal LV neurons relate to the two sublayers, $\mathrm{LVa}$ and $\mathrm{LVb}$, was unclear. Here, we examined this by analysing a total of 121 neurons recorded from the TG mouse line (MEC-13-53D): 31 LEC-LVa, 45 LEC-LVb, 20 MEC-LVa, and 25 MEC-LVb neurons (Figure 2A). As previously reported (Canto and Witter, 2012a, 2012b; Hamam et al., 2000, 2002), only a few LV neurons showed weak depolarizing afterpotentials (DAP; Figure 2B), with a higher incidence in MEC than in LEC. Among the twelve examined electrophysiological properties (Figure 2-figure supplement 1), differences were observed between the $\mathrm{LVa}$ and $\mathrm{LVb}$ neurons in most parameters except for resting potential, input resistance and action potential (AP) threshold (Figure 2F-K, Figure 2 -figure supplement 2). Principal component analysis based on the twelve parameters resulted in a clear separation between $\mathrm{LVa}$ and $\mathrm{LVb}$ neurons, and also in a moderate separation between LEC-LVb and MEC-LVb (Figure 2L). Sag ratio (Figure 2C, F), time constant (Figure 2G), and AP frequency after 200 pA injection (Figure 2E, H) were the three prominent parameters which separated LVa and LVb neurons (Figure $2 \mathrm{M}$ ) with the sag ratio and AP frequency after $200 \mathrm{pA}$ injection being smaller in LVa than LVb, and the opposite was true for the time constant. The difference in sag ratio may indicate that $\mathrm{LVb}$ neurons show more prominent subthreshold oscillations, which have been reported to occur in LV, although details on differences between the two sublayers have not been studied (Egorov et al., 2002; Schmitz et al., 1998). The clearest features aiding in separating LEC-LVb and MEC-LVb were time constant (Figure 2G), AP frequency after 200 pA injection (Figure 2E, $\mathrm{H}$ ), and fast afterhyperpolarization (AHP; Figure 2I, N). Neurons in MEC-LVb showed a smaller time constant, higher AP frequency, and smaller fast AHP than LEC-LVb neurons. Although LVb neurons in LEC and MEC thus differed in some of their electrophysiological characteristics, as well as morphologically (described above; Figure 1G-J), it remains to be determined how these two features influence neuronal and network activity. 


\section{Local projections of $L V b$ neurons in LEC and MEC are different}

187 Subsequently, we examined the local entorhinal LVb circuits, by injecting a tTA-dependent AAV carrying both the channelrhodopsin variant oChiEF and the yellow fluorescent protein (citrine, AAV2/1-TRE-Tight-oChIEF-citrine) into the deep layers of either LEC or MEC in mouse line MEC-13-53D (Figure 3A). This enabled specific expression of the fused oChIEFcitrine protein in either LEC-LVb (Figure 3B-F) or MEC-LVb (Figure 3G-K). Not only the dendrites and the soma but also the axons of these $\mathrm{LVb}$ neurons were clearly labelled. As shown in the horizontal sections taken at different dorsoventral level (Figure 3B-D, G-I), citrine-labelled axons were observed mainly within the entorhinal cortex, and only very sparse labelling was observed in other regions, including the angular bundle, a major efferent pathway of EC. This result supports our previous study (Ohara et al., 2018) showing that the main targets of the entorhinal LVb neurons are neurons in superficially positioned layers. Within EC, the distribution of labelled axons differed between LEC and MEC (Figure 3L). Although in both LEC and MEC, labelled axons were densely present in LIII rather than in layers II and I, we report a striking difference between LEC and MEC in LVa, as is easily appreciated from Figure 3L-M: many labelled axons of LEC-LVb neurons were present in LVa, whereas in case of MEC-LVb, the number of labelled axons was very low in LVa. Such entorhinal labelling patterns were not affected by the unintended labelled neurons in the deep perirhinal cortex (PER; Figure 3D) or postrhinal cortex (POR; Figure 3I), since these neurons hardly project to LEC and MEC (Figure 3-figure supplement 1A-C). It is also very unlikely that the sparse labelling patterns in MEC-LVa is a false negative result due to the selective targetting of a $\mathrm{LVb}$ subpopulation that modestly project to $\mathrm{LVa}$, for two reasons. First, the PCP4-labeling patterns referred to above also differ in LVa between MEC and LEC: PCP4labeled fibers are hardly present in MEC LVa, whereas the axonal density is much higher in LEC LVa (Figure1-figure supplement 1). Second, a strikingly similar labelling pattern was observed in LVa of MEC following an anterograde tracer injection into MEC-LVb in wildtype mice (Figure 3 - figure supplement 1D). Note that we also confirmed this labelling pattern in rat MEC (Figure 3-figure supplement 1E), which is line with a previous study (Köhler, 1986). Based on these anatomical observations, we predicted that LVb neurons in both LEC and MEC innervate LIII neurons rather than LII neurons. Importantly, our findings further indicate that LVb-to-LVa connections, which mediate the hippocampal-cortical output circuit are much more prominent in LEC than in MEC. To test these predicted connectivity patterns, we used optogenetic stimulation of the oChIEF-labelled axons together with patchclamp recordings of neurons in the different layers of EC.

\section{Translaminar local connections of MEC-LVb neurons}

We first examined the $\mathrm{LVb}$ circuits in MEC, by performing patch-clamp recording from principal neurons in layers II ( $\mathrm{n}=20$ for stellate cells, $\mathrm{n}=18$ for pyramidal cells), III ( $\mathrm{n}=30)$, and $\mathrm{Va}(\mathrm{n}=18)$, while optically stimulating $\mathrm{LVb}$ fibers in acute horizontal entorhinal slices (Figure 4, Figure 4-figure supplement 1). Recorded neurons were labelled with biocytin, and the neurons were subsequently defined from the location of their cell bodies, morphological characteristics, and electrophysiological properties. In line with previous studies, LIII principal neurons were pyramidal cells, while neurons in LII were either stellate cells or pyramidal neurons (Figure 4A) (Canto and Witter, 2012a; Fuchs et al., 2016; Winterer et al., 2017). LII stellate cells were not only identified by the morphological features but also from their unique physiological properties, characterized by the pronounced sag potential and DAP (Figure 4B) (Alonso and Klink, 1993).

There was a densely labelled axonal plexus in LIII, which is the layer where LIII pyramidal neurons mainly distribute their basal dendrites. In line with this anatomical observation, all 
LIII neurons (30 out of 30 cells) responded to the optical stimulation (Figure 4C, D). In contrast, the axonal labelling was sparse in LII, and this distribution was reflected in the observed sparser connectivity. The percentage of pyramidal neurons in LII responding to optical stimulation was $61.1 \%$ (11 out of 18 cells) and this percentage was especially low in stellate cells ( $25.0 \% ; 5$ out of 20 cells). Even in the five stellate cells that responded to the light stimulation, evoked responses were relatively small as measured by the amplitude of the synaptic event (Figure 4C, E, Figure 4-figure supplement 1C, E. In order to compare the differences of excitatory postsynaptic potential (EPSP) amplitudes across different layers/cell types, the voltage responses of each neuron were normalized to the response of LIII cells recorded in the same slice (Figure 4F). The normalized EPSP amplitude of LII cells were significantly smaller than those of LIII pyramidal cells, and within LII cells, the normalized responses of stellate cells were significantly smaller than those of pyramidal cells (LII stellate cells, $0.24 \pm 0.06$; LII pyramidal cells, $0.63 \pm 0.08$; LIII pyramidal cells, $1.0 \pm 0.03 ; p<0.001$ for LIIs vs LIII and LIIp vs LIII, $\mathrm{p}<0.01$ for LIIs vs LIIp, One-way ANOVA followed by Bonferroni's multiple comparison test). This difference in responses between LII and III neurons is likely due to the difference of the $\mathrm{LVb}$ fiber distribution within these layers. In contrast, the difference of responses between the two cell types in LII might be explained by the difference of the input resistance between these neurons, the input resistances of stellate cells being significantly lower than that of pyramidal cells $(101.2 \pm 5.9$ versus $278.9 \pm 29.1$ $\mathrm{M} \Omega ; \mathrm{p}<0.001$, One-way ANOVA followed by Bonferroni's multiple comparison test; Figure 4G), although we cannot exclude a possible difference in total synaptic input between the stellate and pyramidal neurons in LII. Note that the latency of the EPSP onset for LII stellate cells was significantly longer than that for LIII cells $(4.3 \pm 0.7$ versus $2.9 \pm 0.1 \mathrm{~ms} ; \mathrm{p}$ $<0.5$, One-way ANOVA followed by Bonferroni's multiple comparison test; Figure $4 \mathrm{H})$. In contrast, the inputs to LII pyramidal cells showed latencies similar to those of LIII cells (3.2 \pm 0.2 versus $2.9 \pm 0.1 \mathrm{~ms}$ ). These differences in latencies indicate that projections from $\mathrm{LVb}$ to LIII and LII pyramidals may be monosynaptic and those to LII neurons may be disynaptic. Since we did not assess this pharmacologically, it is hard to conclude, in particular since in a parallel study in mouse LEC LII, using similar viral and laser stimulation protocols, we measured latencies ranging from $4.5-6.8 \mathrm{msec}$, which consistently were shown to be monosynaptic when tested using pharmacological measures (Nilssen, 2019).

As shown in Figure 1-figure supplement 4, and also in line with previous studies (Canto and Witter, 2012a; Hamam et al., 2000; Sürmeli et al., 2015), LVa pyramidal cells have their basal dendrites mainly confined to LVa, which is the layer that MEC-LVb neurons avoid to project to (Figure 3L, Figure 4A, Figure 4-figure supplement 1D, G). In line with this anatomical observation, only $27.8 \%$ (5 out of 18 cells) responded to the light stimulation (Figure 4D). The EPSP amplitudes of the responding LVa neurons were relatively small (Figure 4C, E, Figure 4-figure supplement 1F, H), and the normalized EPSPs were significantly smaller than those of LIII neurons $(0.47 \pm 0.10$ versus $1.0 \pm 0.03 ; p<0.001$, One-way ANOVA followed by Bonferroni's multiple comparison test; Figure 4F). In addition, the latency of the EPSP onset for LV a cells was significantly longer than that for LIII cells $(5.6 \pm 1.7$ versus $2.9 \pm 0.1 \mathrm{~ms} ; \mathrm{p}<0.001$, One-way ANOVA followed by Bonferroni's multiple comparison test; Figure $4 \mathrm{H}$ ) indicating that these responses are either the result of monosynatic inputs onto the apical dendrite in LIII or that they represent disynaptic responses.

We recorded in slices taken at different dorsoventral levels. Since functional differences along this axis has been reported (Steffenach et al., 2005; Stensola et al., 2012), we examined whether the LVb-LVa connectivity differs along the dorsoventral axis, by grouping the recorded LVa responses in three distinct dorsoventral levels (Figure 4-figure supplement 2). 
The voltage responses of the more ventrally positioned LVa neurons were significantly higher than those measured more dorsally in MEC $(\mathrm{p}<0.01$, One-way ANOVA followed by Bonferroni's multiple comparison test). Since the EPSP amplitudes of LIII neurons did not differ at different dorsoventral levels, it is unlikely that the observed response differences are caused by different levels of oChiEF-expression in LVb fibers along the dorsoventral axis. The observed difference may be caused by the difference in severing of apical dendrites at different dorsoventral levels, since the axons of MEC-LVb neurons massively distribute in LIII. This, however, does not seem to be the case since nine out of ten LVa-neurons with intact/substantial amount of apical dendrites $(>150 \mu \mathrm{m})$ in LIII did not respond to the light stimulation.

\section{Translaminar local connections of LEC-LVb neurons}

We next examined the LVb local circuits in LEC with the similar method as applied in MEC (above). In LEC, LII can further be divided into two sublayers: a superficial layer IIa (LIIa) composed of fan cells, and a deep layer IIb ( $\mathrm{LIIb}$ ) mainly composed of pyramidal neurons (Leitner et al., 2016). Fan cells mainly extend their apical dendrites in LI, where the density of LVb labelled-fibers is extremely low (Figure 5A, Figure 5-figure supplement 1). This contrasts with LIIb, LIII, and LVa neurons, which distribute at least part of their dendrites in layers with a relatively high density of $\mathrm{LVb}$ axons. In line with these anatomical observations, only $26.9 \%$ of the fan cells (7 out of 26 neurons) responded to the light stimulation (Figure $5 \mathrm{C}, \mathrm{D})$. On the other hand, the response probabilities of LIIb, III, and Va were high, $76.9 \%$ (20 out of 26 cells), $100 \%$ (34 out of 34 cells), and $94.7 \%$ (18 out of 19 cells), respectively. The voltage responses of these neurons were also significantly larger than those of the LIIa neurons (LIIa neurons, $0.15 \pm 0.03$; LIIb neurons, $0.79 \pm 0.15$; LIII neurons, $1.0 \pm 0.04$; LVa neurons, $0.93 \pm 0.10 ; \mathrm{p}<0.01$ for LIIa vs LIIb, $\mathrm{p}<0.001$ for LIIa vs LIII and LIIa vs LVa, One-way ANOVA followed by Bonferroni's multiple comparison test; Figure 5E, F). In contrast to MEC-LII stellate cells, the input resistance of LIIa fan cells was significantly higher than that of LIIb and LIII neurons (LIIa neurons, $323.6 \pm 16.1$; LIIb neurons, $226.5 \pm$ 17.2; LIII neurons, $194.3 \pm 13.3 \mathrm{M} \Omega$; $\mathrm{p}<0.001$, One-way ANOVA followed by Bonferroni's multiple comparison test; Figure $5 \mathrm{G}$ ). This indicates that the small responses of LIIa fan cells cannot be explained by the differences in input resistance among the superficial neurons and may simply be due to the small number of synaptic inputs to LIIa fan cells from LVb neurons. In contrast to MEC, LEC-LVa neurons showed large responses to light stimulation, which matched with the anatomically dense LVb fiber distribution in LEC-LVa (Figure 3L). In line with our previous monosynaptic input tracing study using rabies virus (Ohara et al., 2018), the latency of the EPSP onset, which was similar in all cell types (LIIa neurons, $4.8 \pm 1.1$; LIIb neurons, $5.0 \pm 1.4$; LIII neurons, $4.2 \pm 0.9$; LVa neurons, $4.2 \pm 0.6 \mathrm{~ms}$; Figure $5 \mathrm{H}$ ), points to the LVb-to-LVa connectivity, as well as that to LIII and LII, being largely monosynaptic.

The striking difference between MEC and LEC regarding LVb to LVa projections is clear from comparing the proportion of responding neurons (Figure 5I), and the normalized EPSP based on LIII response (Figure 5J) between MEC and LEC. In contrast to the similar responses of LII neurons between the two subregions, the normalized voltage responses of LEC-LVa neurons were significantly larger than those of MEC-LVa neurons $(0.93 \pm 0.10 \mathrm{vs}$ $0.47 \pm 0.10, p<0.05$, two-tailed unpaired t-test; Figure $5 \mathrm{~J})$. On a final note, it is apparent that postsynaptic responses in LEC are larger than those in MEC. This difference in response amplitudes is most likely due to the differences in the number of oChiEF-expressing neurons since, as shown in Figure 1E, the proportion of tTA-expressing neurons is higher in LEC than in MEC. We deem it unlikely that these amplitude differences are caused by differences in 
biophysical properties since no such differences have been reported between matching cell types in LEC and MEC (Canto and Witter, 2012a).

The present data clearly show that neurons in LVb of both LEC and MEC give rise to dense intrinsic projections to more superficial layers and show laminar preferences (Figure 6). We noticed a striking difference between the two entorhinal regions, in that neurons in LEC-LVb innervated LVa neurons, whereas in dorsal MEC this was rarely observed. In contrast, other intrinsic circuits from LVb to LII/III were very similar in both entorhinal subdivisions, which preferentially targeted pyramidal cells rather than the stellate or fan cells in LII. These data indicate that the lack of experimental support for an effective LVb-to-LVa projection in MEC is not due to technical issues and thus supports our conclusion that the intrinsic LV circuitry underlying the canonical hippocampal-cortical output circuit is poorly developed in dorsal MEC.

\section{Discussion}

In this study, we experimentally tested the major assumption about the organization of hippocampal-cortical output circuits via entorhinal LVb neurons, considered to be crucial for the normal functioning of the medial temporal lobe memory system, more in particular systems memory consolidation. Our key finding is that LEC and MEC are strikingly different with respect to the hippocampal-cortical pathway mediated by LV neurons, in that we obtained electrophysiological evidence that this postulated crucial circuit is more effective in LEC than in MEC. In addition, we present new data that point to three major functionally relevant insights in the organization of the intrinsic translaminar entorhinal network originating from $\mathrm{LVb}$ neurons.

First, the present data indicate that LVb pyramidal neurons in LEC and MEC differ with respect to main morphological and electrophysiological characteristics. In contrast, LVa neurons in MEC and LEC are rather similar in these two aspects. Second, we show that projections from principal neurons in $\mathrm{LVb}$ in both entorhinal subdivisions preferentially synapse onto pyramidal neurons in LIII and LII. LVb neurons have a much weaker synaptic relationship with principal neurons in LII that project to the dentate gyrus (DG) and the CA3/CA2 region, i.e. stellate and fan cells. Last, and most important, our data point to a new and challenging circuit difference between the two entorhinal subdivisions with respect to the inputs to LVa neurons, i.e. the output neurons of EC. Whereas in LEC, LVa neurons receive substantial input from $\mathrm{LVb}$ neurons, this projection is apparently weak to absent in dorsal MEC. The weak and sparse LVb-to-LVa projections in dorsal MEC, though unexpected in view of previous data including our own rabies tracing data (Ohara et al., 2018), has been recently corroborated in an in vitro study using paired-patch recording (Rozov et al., 2020), and our present corroborating tracing data are in line with previous tracing data in the rat (Köhler, 1986) and monkey (Chrobak and Amaral, 2007).

\section{Layer Vb neurons in LEC and MEC are morphologically and electrophysiologically different}

The use of layer specific transgenic mice allowed us to differentiate neurons in LVa from those in LVb and to differentiate these layer specific neuron-types between LEC and MEC. This contrasts with previous studies in rats, showing that LV neurons in both LEC and MEC share electrophysiological properties (Canto and Witter, 2012a, 2012b; Hamam et al., 2000, 
2002), although these authors did differentiate between LVa and LVb neurons based on morphological criteria and laminar distribution. We corroborate the reported morphological differences and add that the neurons also differ with respect to their electrophysiological properties. The most striking difference between MEC- and LEC-LVb neurons however is in the morphology of the apical dendrite. Neurons in MEC-LVb have an apical dendrite that heads straight to the pia, such that distal branches reach all the way up into LI, which is in line with previous studies (Canto and Witter, 2012a; Hamam et al., 2000; Sürmeli et al., 2015). In contrast, the apical dendrites of LEC-LVb neurons have a more complex branching pattern and they do not extend beyond LIII. This indicates that LEC-LVb neurons are unlikely to be targeted by inputs to LEC that selectively distribute to layers I and II, such as those carrying olfactory information from the olfactory bulb and the piriform cortex (Luskin and Price, 1983) as well as commissural projections (Leitner et al., 2016). The LVb neurons in LEC are thus dissimilar to their counterparts in MEC which are morphologically suited to receive such superficially terminating inputs, as has been shown for inputs from the parasubiculum (Canto et al., 2012) and contralateral MEC (Fuchs et al., 2016). The here reported differences between $\mathrm{LVb}$ neurons, with MEC-LVb neurons showing a shorter time constant than LEC-LVb neurons, further indicates that MEC-LVb neurons have a shorter time window to integrate inputs compared to LEC-LVb neurons (Canto and Witter, 2012a, 2012b). The differences in AP frequency and half duration of AP may result in differences in the propensity of neurons to show graded persistent firing, which is prominent in MEC LV. Unfortunately, reports of persistent activity in MEC do not differentiate between neurons in LVa and LVb (Egorov et al., 2002; Fransén et al., 2006). However, up-down state activity originating in LIII particularly entrains neurons in LVb (Beed et al., 2020), indicating that indeed $\mathrm{LVb}$ neurons might preferentially show persistent activity. Together, these differences will result in differences in information processing

\section{Layer Vb neurons preferentially target pyramidal neurons in layers III and II and avoid layer II neurons that project to the dentate gyrus.}

Both our anatomical and electrophysiological data show that projections from principal neurons in $\mathrm{LVb}$ in both entorhinal subdivisions preferentially synapse onto pyramidal neurons in LIII and LII. LVb neurons have a much weaker synaptic relationship with the class of stellate and fan cells in MEC or LEC, respectively. This makes it likely that in both LEC and MEC, hippocampal information preferentially interacts with neurons that are part of the LIII-to-CA1/Sub projection system rather than with the LII-to-DG/CA2-3 projecting neurons. Additional target neurons in layer II/III might be the pyramidal neurons that project contralaterally, which in LII belong to the Calbindin (CB+) population (Ohara et al., 2019; Steward and Scoville, 1976; Varga et al., 2010), as well as the substantial population of CB+ excitatory intrinsic projection neurons (Ohara et al., 2019). The present findings are in line with a previous study using wild type mice, reporting that most of the inputs to MEC-LII stellate cell arise from superficial layers, whereas those of MEC-LII pyramidal cells arise from the deep layers (Beed et al., 2010).

The sparse projection from MEC LVb neurons to LII stellate cells and the more massive projection to LII pyramidal cell, was unexpected for two reasons. First, both the stellate and the CB+ population of layer II pyramidal neurons contain grid cells (Hafting et al., 2005; Tang et al., 2014) and hippocampal excitatory inputs are required for the formation and translocation of grid patterns (Bonnevie et al., 2013). Though our data do not exclude that LVb inputs can reach LII stellate cells indirectly through LIII- and LII-pyramidal cells (Ohara et al., 2019; Winterer et al., 2017), they do indicate that the two populations of grid 
cells, stellate versus $\mathrm{CB}+$ cells, might differ with respect to the strength of a main excitatory drive from the hippocampus.

Second, re-entry of hippocampal activity, i.e. the presence of recurrent circuits, have been proposed as one of the mechanisms for temporal storage of information in a neuronal network (Edelman, 1989; Iijima et al., 1996). Re-entry through LII-to-DG has been observed in in vivo recordings under anesthesia in rats, although this was examined with current source density analysis which is not optimal to exclude multisynaptic responses (Kloosterman et al., 2004). Such multisynaptic inputs could be mediated by pyramidal neurons in LIII and LII, both of which do contact layer II-to-DG projecting neurons (Ohara et al., 2019; Winterer et al., 2017). Our current data strongly favour the circuit via LIII-CA1/subiculum in both entorhinal subdivisions to mediate a recurrent hippocampal-entorhinal-hippocampal circuit. The importance of this layer III recurrent network is corroborated by the observation that entorhinal LIII input to the hippocampus field CA1 plays a crucial role in associating temporally discontinuous events and retrieving remote memories (Lux et al., 2016; Suh et al., 2011)

\section{Layer Vb projections to layer Va exert prominent effects in LEC but not in MEC}

Ever since the seminal observation in monkeys and rats of a hippocampal-cortical projection 1977), 1977), the canonical circuit underlying the hippocampal-cortical interplay, necessary for memory consolidation (Buzsáki, 1996; Eichenbaum et al., 2012), is believed to use EC LV neurons that receive hippocampal output and send projections to the neocortex. More recent studies in rats and mice indicated that neurons in LVb likely are the main recipients of this hippocampal output stream (Sürmeli et al., 2015) and that principal neurons in LVa form the main source of outputs to neocortical areas (Ohara et al., 2018; Sürmeli et al., 2015). The very sparse connection from LVb-to-LVa in dorsal MEC reported here thus indicates that at least in dorsal MEC, the canonical role of EC LV neurons to mediate hippocampal information transfer to downstream neocortical areas needs to be revised. In contrast to the dorsal level, we showed at more ventral levels an increased LVb-to-LVa connectivity, in line with our previous study (Ohara et al., 2018). Interestingly, a recent study has reported that there is a direct projection from the intermediate/ventral hippocampus to neurons in MEC LVa (Rozov et al., 2020), indicating that the hippocampal-output pathway via MEC may differ along the dorsoventral axis. Our data in LEC show the presence of strong and effective connections from LVb to LVa, and this striking difference with MEC is also reflected in the observation that LVa, the entorhinal-output layer, is thicker in LEC than in MEC. Together, this suggests that LEC might be the more relevant player in mediating the hippocampalcortical interplay relevant for systems memory consolidation (Buzsáki, 1996; Eichenbaum et al., 2012; Frankland and Bontempi, 2005). However, studies which have functionally linked the LVa-output projection with memory consolidation are based on data obtained in MEC (Kitamura et al., 2017). This more likely reflects the strong focus on functions of MEC circuits rather than LEC circuits ever since the discovery of the grid cell (Hafting et al., 2005; Moser et al., 2017). With the discovery of LEC networks coding for event sequences (Bellmund et al., 2019; Montchal et al., 2019; Tsao et al., 2018), this is likely to change.

\section{Conclusion}

467 Our experimental observations lead us to suggest that LEC might be more relevant than MEC 468 in mediating the export of information from the dorsal hippocampus to the neocortex, a projection that allegedly is crucial to systems memory consolidation. In contrast, the two entorhinal subdivisions share the connectional motifs underlying transfer of hippocampal 
output back to the hippocampal formation, thus mediating re-entry. Finally, our data indicate that this re-entry circuit preferentially uses pyramidal neurons projecting to CA1 and subiculum and much less interacts with neurons projecting to the dentate gyrus and CA3/CA2.

\section{Methods}

\section{Animals}

All animals were group housed at a 12:12 h reversed day/night cycle and had ad libitum access to food and water. Mice of the transgenic MEC13-53D enhancer strain expressing tetracycline transactivator (tTA) in PCP4-positive entorhinal LVb neurons (Blankvoort et al., 2018) were used for whole-cell recordings $(n=38)$, and for histological assessment of specific transgene expression $(n=7)$. To characterize the tTA expression patterns in this mouse line, MEC13-53D was crossed with a tetO-GCaMP6-mCherry line (Blankvoort et al., 2018; $n=2$ ). Other transgenic mouse lines, GAD67-GFP (Tanaka et al., 2003; n=4) and Rosa26TdTomatoAi9 (Madisen et al., 2010; $n=2$ ), were used to characterize entorhinal neurons in layer $\mathrm{Va}$ and $\mathrm{Vb}$. We further used C57BL/6N mice to characterize the morphology of entorhinal LVa neurons ( $\mathrm{n}=2$ ), and to examine the projection of entorhinal $\mathrm{LVb}$ neurons and PER neurons in wild-type mice $(n=2)$. The projection of PER/POR neurons were also examined in MEC13-53D ( $\mathrm{n}=2)$. All experiments were approved by the local ethics committee and were in accordance with the European Communities Council Directive and the Norwegian Experiments on Animals Act.

\section{Surgical Procedures and Virus/Tracer Injections}

Animals were anesthetized with isoflurane in an induction chamber (4\%, Nycomed, airflow 1 $\mathrm{L} / \mathrm{min}$ ), after which they were moved to a surgical mask on a stereotactic frame (Kopf Instruments). The animals were placed on a heating pad $\left(37^{\circ} \mathrm{C}\right)$ to maintain stable body temperature throughout the surgery, and eye ointment was applied to the eyes of the animal to protect the corneas from drying out. The animals were injected subcutaneously with buprenorphine hydrochloride $(0.1 \mathrm{mg} / \mathrm{kg}$, Temgesic, Indivior), meloxicam $(1 \mathrm{mg} / \mathrm{kg}$, Metacam Boehringer Ingelheim Vetmedica), and bupivacaine hydrochloride (Marcain 1 $\mathrm{mg} / \mathrm{kg}$, Astra Zeneca), the latter at the incision site. The head was fixed to the stereotaxic frame with ear bars, and the skin overlying the skull at the incision site was disinfected with ethanol $(70 \%)$ and iodide before a rostrocaudal incision was made. A craniotomy was made around the approximate coordinate for the injection, and precise measurements were made with the glass capillary used for the virus injection. The coordinates of the injection sites are as follows (anterior to either bregma (APb) or transverse sinus (APt), lateral to sagittal sinus (ML), ventral to dura (DV) in mm): LEC (APt +2.0, ML 3.9, DV 3.0), MEC (APt +1.0, ML 3.3, DV 2.0), NAc (APb +1.2, ML 1.0, DV 3.8), RSC (APb -3.0, ML 0.3, DV 0.8), PER (APb -4.5, ML 4.5, DV 1.5), POR (APt +1.1, ML 3.3, DV 0.9). Viruses were injected with a nanoliter injector (Nanoliter 2010, World Precision Instruments) controlled by a microsyringe pump controller (Micro4 pump, World Precision Instruments); 100-300 nl of virus was injected with a speed of $25 \mathrm{nl} / \mathrm{min}$. The capillary was left in place for an additional $10 \mathrm{~min}$ after the injection, before it was slowly withdrawn from the brain. Finally, the wound was rinsed, and the skin was sutured. The animals were left to recover in a heating chamber, before being returned to their home cage, where their health was checked daily.

For electrophysiological studies, young MEC13-53D mice (5 - 7 weeks old) were injected with a tTA-dependent adeno-associated virus (AAV, serotype 2/1) carrying either green fluorescent protein (GFP) or a fused protein of oChIEF, a variant of the light-activating 
protein channelrhodopsin2 (Lin et al., 2009), and citrine, a yellow fluorescent protein (Griesbeck et al., 2001). The construction of these virus, AAV-TRE-tight-GFP and AAVTRE-tight-oChIEF-Citrine respectively, has been described in Nilssen et al (2018). These samples were also used to characterize the transgenic mouse line and also the projection patterns of entorhinal LVb neurons. To label LVa neurons, retrograde AAV expressing enhanced blue fluorescent protein (EBFP) and Cre recombinase (AAVrg-pmSyn1-EBFP-cre, Addgene \#51507) was injected into either NAc or RSC of Rosa26TdTomatoAi9. LVa neurons were also labelled in C57BL/6N mice, by injecting AAVrg-pmSyn1-EBFP-cre in NAc while injecting AAV-CMV-FLEX-mCherry in LEC/MEC. The pAAV-FLEX-mCherryWPRE construct was created by first cloning a FLEX cassette with MCS into Cla1 and HindIII sites in pAAV-CMV-MCS-WPRE (Agilent) to create pAAV-CMV-FLEX-MCSWPRE. The sequence of the FLEX cassette was obtained from Atasoy et al (2008). Subsequently, the mCherry sequence was synthesized and cloned in an inverted orientation into EcoR1 and BamH1 sites in pAAV-CMV-FLEX-MCS-WPRE to make pAAV CMVFLEX-mCherry-WPRE. AAV-CMV-FLEX-mCherry was recovered from pAAV CMVFLEX-mCherry-WPRE as described elsewhere (Nair et al., 2020; Nilssen et al., 2018).

For anterograde tracing experiments in wild-type animals, either $2.5 \%$ Phaseolus vulgarisleucoagglutinin (PHA-L; Vector Laboratories) or 3.5\% $10 \mathrm{kDa}$ biotinylated dextran amine (BDA, Invitrogen, Molecular Probes) was injected iontophoretically with positive $6 \mu \mathrm{A}$ current pulses ( $6 \mathrm{~s}$ on; $6 \mathrm{~s}$ off) for $15 \mathrm{~min}$. To label projections from PER in C57BL/6N mice, AAV1.CAG.tdTomato.WPRE.SV40 (Upenn viral core, Cat. AV-1-PV3365) was injected iontophoretically with positive $5 \mu \mathrm{A}$ current pulses ( $5 \mathrm{~s}$ on; $5 \mathrm{~s}$ off) for $5 \mathrm{~min}$.

\section{Acute slice preparation}

Two to three weeks after AAV injection, acute slice preparations were prepared as described in detail (Nilssen et al., 2018). Briefly, mice were deeply anesthetized with isoflurane and decapitated. The brain was quickly removed and immersed in cold $\left(0^{\circ} \mathrm{C}\right)$ oxygenated $(95 \%$ $\mathrm{O}_{2} / 5 \% \mathrm{CO}_{2}$ ) artificial cerebrospinal fluid (ACSF) containing $110 \mathrm{mM}$ choline chloride, 2.5 $\mathrm{mM} \mathrm{KCl}, 25 \mathrm{mM}$ D-glucose, $25 \mathrm{mM} \mathrm{NaHCO} 3,11.5 \mathrm{mM}$ sodium ascorbate, $3 \mathrm{mM}$ sodium pyruvate, $1.25 \mathrm{mM} \mathrm{NaH}_{2} \mathrm{PO}_{4}, 100 \mathrm{mM}$ D-mannitol, $7 \mathrm{mM} \mathrm{MgCl}_{2}$, and $0.5 \mathrm{mM} \mathrm{CaCl}_{2}, \mathrm{pH} 7.4$, $430 \mathrm{mOsm}$. The brain hemispheres were subsequently separated and $350 \mu \mathrm{m}$ thick entorhinal slices were cut with a vibrating slicer (Leica VT1000S, Leica Biosystems). We used semicoronal slices for LEC recording, which were cut with an angle of $20^{\circ}$ with respect to the coronal plane to optimally preserve neurons and local connections of LEC (Canto and Witter, 2012b; Nilssen et al., 2018). In case of MEC recordings, horizontal slices were prepared (Canto and Witter, 2012a; Couey et al., 2013). Slices were incubated in a holding chamber at $35^{\circ} \mathrm{C}$ in oxygenated $\mathrm{ASCF}$ containing $126 \mathrm{mM} \mathrm{NaCl}, 3 \mathrm{mM} \mathrm{KCl}, 1.2 \mathrm{mM} \mathrm{Na}_{2} \mathrm{HPO}_{4}, 10 \mathrm{mM}$ D-glucose, $26 \mathrm{mM} \mathrm{NaHCO}_{3}, 3 \mathrm{mM} \mathrm{MgCl}$, and $0.5 \mathrm{mM} \mathrm{CaCl}_{2}$ for $30 \mathrm{~min}$ and then kept at room temperature for at least $30 \mathrm{~min}$ before use.

\section{Electrophysiological recording}

Patch clamp recording pipettes (resistance:4-9 M $\Omega$ ) were made from borosilicate glass capillaries (1.5 outer diameter x 0.86 inner diameter; Harvard Apparatus) and back-filled with internal solution of the following composition: $120 \mathrm{mM} \mathrm{K}$-gluconate, $10 \mathrm{mM} \mathrm{KCL}, 10 \mathrm{mM}$ $\mathrm{Na}_{2}$-phosphocreatine, $10 \mathrm{mM}$ HEPES, $4 \mathrm{mM} \mathrm{Mg-ATP}, 0.3 \mathrm{mM} \mathrm{Na}-\mathrm{GTP}$, with pH adjusted to 7.3 and osmolality to $300-305 \mathrm{mOsm}$. Biocytin ( $5 \mathrm{mg} / \mathrm{mL}$; Iris Biotech) was added to the internal solution in order to recover cell morphology. Acute slices were moved to the recording setup and visualized using infrared differential interference contrast optics aided by a 20x/1.0 NA water immersion objective (Zeiss Axio Examiner D1, Carl Zeiss). 
Electrophysiological recordings were performed at $35^{\circ} \mathrm{C}$ and slices superfused with oxygenated recording ACSF containing $126 \mathrm{mM} \mathrm{NaCl}, 3 \mathrm{mM} \mathrm{KCl}, 1.2 \mathrm{mM} \mathrm{Na}_{2} \mathrm{HPO}_{4}, 10$ $\mathrm{mM}$ D-Glucose, $26 \mathrm{mM} \mathrm{NaHCO}_{3}, 1.5 \mathrm{mM} \mathrm{MgCl}_{2}$ and $1.6 \mathrm{mM} \mathrm{CaCl}_{2}$. $\mathrm{LVb}$ in both LEC and MEC was identified through the presence of the densely packed small cells, and LVb neurons labelled with GFP were selected for recording. LVa neurons were selected for recording on the basis of their large soma size and the fact that they are sparsely distributed directly superficial to the smaller neurons of LVb. Gigaohm resistance seals were acquired for all cells before rupturing the membrane to enter whole-cell mode. Pipette capacitance compensation was performed prior to entering whole-cell configuration, and bridge balance adjustments were carried out at the start of current clamp recordings. Data acquisition was performed by Patchmaster (Heka Elektronik) controlling an EPC 10 Quadro USB amplifier (Heka Elektronik). Acquired data were low-pass Bessel filtered at $15.34 \mathrm{kHz}$ (for whole-cell current-clamp recording) or $4 \mathrm{kHz}$ (for whole-cell voltage-clamp recording) and digitized at $10 \mathrm{kHz}$. No correction was made for the liquid junction potential $(13 \mathrm{mV}$ as measured experimentally). Data were discarded if the resting membrane potential was $\geq-57 \mathrm{mV}$ or/and the series resistance was $\geq 40 \mathrm{M} \Omega$.

Intrinsic membrane properties were measured from membrane voltage responses to step injections of hyperpolarizing and depolarizing current ( $1 \mathrm{~s}$ duration, $-200 \mathrm{pA}$ to $200 \mathrm{pA}, 20$ pA increments). Acquired data were exported to text file with MATLAB (The MathWorks) and were analysed with Clampfit (Molecular Devices). The following electrophysiological parameters analysed were defined as follows:

Resting membrane potential $\left(\mathrm{V}_{\text {rest }} ; \mathrm{mV}\right)$ : membrane potential measured with no current applied ( $\mathrm{I}=0$ mode);

Input resistance (Mohm): resistance measured from Ohm's law from the peak of voltage responses to hyperpolarizing current injections (-40 pA injection);

Time constant (ms): the time it took the voltage deflection to reach $63 \%$ of peak of voltage response at hyperpolarizing current injections ( $-40 \mathrm{pA}$ injection);

Sag ratio (steady-state/peak): measured from voltage responses to hyperpolarizing current injections with peaks at $-90 \pm 5 \mathrm{mV}$, as the ratio between the voltage at steady-state and the voltage at the peak;

Action potential (AP) threshold $(\mathrm{mV})$ : the membrane potential where the rise of the action potential was $20 \mathrm{mV} / \mathrm{ms}$;

AP peak $(\mathrm{mV})$ : voltage difference from AP threshold to peak;

AP half-width (ms): duration of the AP at half-amplitude from AP threshold;

AP maximum rate of rise $(\mathrm{mV} / \mathrm{ms})$ : maximal voltage slope during the upstroke of the AP;

Fast afterhyperpolarization (fast AHP; in $\mathrm{mV}$ ): the peak of AHP in a time window of $6 \mathrm{~ms}$ after the membrane potential reached $0 \mathrm{mV}$ during the repolarization phase of AP;

Medium AHP (mV): the peak of AHP in a time window of 200ms after fast AHP;

AP frequency after 200pA inj. (Hz): frequency of APs evoked with +200 pA of 1-s-long current injection;

Adaptation: measured from trains of $10 \pm 1$ APs as [1 -(Ffirst/Flast)], where Ffirst and Flast are, respectively, the frequencies of the first and last interspike interval. 
Depolarizing afterpotential (DAP): depolarizing voltage deflection after AP. DAP was defined based on previous studies (Canto and Witter, 2012a, 2012b; Hamam et al., 2000, 2002).

\section{Optogenetic stimulation and patch-clamp data analysis}

oChIEF+ fibers were photostimulated with a $473 \mathrm{~nm}$ laser controlled by a UGA-42 GEO point scanning system (Rapp OptoElectronic), and delivered through a 20x/1.0 NA WI objective (Carl Zeiss Axio Examiner.D1). Laser pulses had a beam diameter of $36 \mu \mathrm{m}$ and a duration of $1 \mathrm{~ms}$. The tissue was illuminated with individual pulses at a rate of $1 \mathrm{~Hz}$ in a $4 \times 5$ grid pattern. The grid was positioned such as to allow for the light stimulation to cover across layer I to Va. Laser power was fixed to an intensity which evokes inward currents (EPSCs) but not action potentials. The voltage- or current-traces from individual stimulation spots were averaged over 4-6 individual sweeps to create an average response for each point in the $4 \times 5$ grid. The stimulation point which showed the largest voltage response was used for further analysis. Deflections of the average voltage trace exceeding 10 SDs ( \pm 10 SDs) of the baseline were classified as synaptic responses. Postsynaptic potentials were calculated as the difference between the peak of the evoked synaptic potential and the baseline potential measured before stimulus onset. The latency of optical activation was defined as the time interval between light onset and the point where the voltage trace exceeded $10 \%$ amplitude. Data analysis was performed using MATLAB. Only slices with at least one successful synaptic response to photostimulation were included in the analysis. There were no outliers which were removed from the data.

All data presented in the figures are shown as mean \pm standard errors. Prism software was used for data analysis (Graphpad software), and one-way ANOVA with Bonferroni's multiple comparison test was used to compare the electrophysiological properties and voltage responses between each cell types. To analyze mediolateral gradient in LVb-to-LVa connectivity of MEC, we used Pearson correlation coefficient. A principal component analysis based on the 12 electrophysiological properties (Figure 2-figure supplement 1) was conducted in MATLAB. For this purpose, all variables were normalized to a standard deviation of 1 .

\section{Histology, Immunohistochemistry, and imaging of electrophysiological slices}

After electrophysiological recordings, the brain slices were put in $4 \%$ paraformaldehyde (PFA, Merck Chemicals) in $0.1 \mathrm{M}$ phosphate buffer (PB) for $48 \mathrm{~h}$ at $4{ }^{\circ} \mathrm{C}$. Slices were permeabilized $5 \times 15$ min in phosphate buffered saline containing $0.3 \%$ Triton X-100 (PBSTx), and were immersed in a blocking solution containing PBS-TX and 10\% Normal Goat Serum (NGS, Abcam: AB7481) for three hours at room temperature. To visualized targeted entorhinal LVb neurons expressing either GFP or oChIEF-citrine, slices were incubated with a primary antibody, chicken anti-GFP (1:500, Abcam), diluted in the blocking solution for 4 days at $4^{\circ} \mathrm{C}$. Note that citrine is derived from Aequorea victoria GFP (Griesbeck et al., 2001), and thus, the signal can be amplified with GFP antibodies. After this, the sections were washed $5 \times 15$ min in PBS-Tx at room temperature and incubated in a secondary antibody, goat anti-chicken (1:400, Alexa Fluor 488, Thermo Fisher Scientific) overnight at room temperature. This secondary antibody incubation was accompanied with the fluorescent conjugated streptavidin (1:600, AF546, Thermo Fisher Scientific) and Neurotrace 640/660 deep-red fluorescent nissl stain (1:200, Thermo Fisher Scientific) in order to stain cells filled with biocytin and to identify the cytoarchitecture. Slices were rinsed in PBS-TX $(3 \times 10 \mathrm{~min})$ at and dehydrated by increasing ethanol concentrations (30\%, 50\%, 70\%, 90\%, 100\%, 100\%, 
10 min each). They were treated to a 1:1 mixture of $100 \%$ ethanol and methyl salicylate for 10 minutes before clearing and storage in methyl salicylate (VWR Chemicals).

To image the recorded neurons with a laser scanning confocal microscope (Zeiss LSM 880 AxioImager Z2), the slices were mounted in custom-made metal well slides with methyl salicylate and coverslipped. Overview images of the tissue were taken at low magnification (Plan-Apochromat 10×, NA 0.45) to confirm the location of the recorded neurons, and at higher magnification (Plan-Apochromat 20x, NA 0.8) to determine the morphology of the recorded neurons. Both overview images and high-magnification images were obtained as $\mathrm{z}$ stacks that included the whole extent of each recorded cell to recover the full cell morphology. The morphology of LVb neurons of MEC/LEC was classified based on previous studies (Canto and Witter, 2012a, 2012b; Hamam et al., 2000, 2002).

\section{Histology, immunohistochemistry, and imaging of neuroanatomical tracing samples}

After two to three weeks of survival, virus- or tracer-injected mice were anesthetized with isoflurane before being euthanized with a lethal intraperitoneal injection of pentobarbital (100 $\mathrm{mg} / \mathrm{kg}$, Apotekerforeningen). They were subsequently transcardially perfused using a peristaltic pump (World Precision Instruments), first with Ringer's solution $(0.85 \% \mathrm{NaCl}$, $0.025 \% \mathrm{KCl}, 0.02 \% \mathrm{NaHCO} 3)$ and subsequently with freshly prepared $4 \%$ PFA in $0.1 \mathrm{M} \mathrm{PB}$ ( $\mathrm{pH}$ 7.4). The brains were removed from the skull, postfixed in PFA overnight, and put in a cryo-protective solution containing $20 \%$ glycerol, $2 \%$ DMSO diluted in $0.125 \mathrm{~m} \mathrm{~PB}$. A freezing microtome was used to cut the brains into $40-\mu \mathrm{m}$-thick sections, that were collected in six equally spaced series for processing.

To enhance the GFP/citrine signal in AAV-TRE-tight-GFP/oChIEF-Citrine infected entorhinal LVb neurons and in GAD67-positive neurons, sections were stained with primary (1:400, chicken anti-GFP, Abcam \#ab13970; 1:2000, rabbit anti-GFP, Thermo Fisher Scientific \#A11122) and secondary antibodies (1:400, AlexaFluor-488 goat anti-chicken IgG, Thermo Fisher Scientific \#A11039; 1:400, Alexa Fluor-546 goat anti-rabbit IgG, Thermo Fisher Scientific \#A11010). This amplification of GFP/citrine-signal, enabled us to examine the axonal distribution of the infected LVb neurons. Due to the strong neurites labelling, however, it became difficult to visualize cell bodies of the targeted neurons. For such reason, we occasionally used the native fluorophore (GFP/citrine) to visualize the cell bodies, while using immunolabeling (anti-GFP/citrine (anti-GFP)) to identify the neurites.

To identify the LVb border and to characterize the transgenic mouse line, LVb neurons were visualized with primary (1:300, rabbit anti-PCP4, Sigma Aldrich \#HPA005792; 1:3000, rat anti-Ctip, Abcam \#ab18465) and secondary antibodies (1:400, Alexa Fluor 633 goat anti-rat IgG, Thermo Fisher Scientific \# A21094; 1:400, Alexa Fluor-546 goat anti-rabbit IgG; 1:400, Alexa Fluor 635 goat anti-rabbit IgG, Thermo Fisher Scientific \# A31576). For delineation purpose, sections were stained with primary (1:1000, guinea pig anti-NeuN, Millipore \#ABN90P; 1:1000, mouse anti-NeuN, Millipore \#MAB377) and secondary antibodies (1:400, Alexa Fluor 647 goat anti-guinea pig IgG, Thermo Fisher Scientific \#A21450; 1:400, Alexa Fluor 488 goat anti-guinea pig IgG, Thermo Fisher Scientific \#A11073; 1:400, Alexa Fluor 488 goat anti-mouse IgG, Thermo Fisher Scientific \#A11001). PHA-L was visualized with primary (1:1000, goat anti-PHA-L, Vector Laboratories L-1110) and secondary antibodies (1:400, Alexa Fluor 635 goat anti-rabbit IgG), while BDA was visualized with Cy3streptavidin (1:400, Jackson ImmunoResearch 016-160-084).

For all immunohistochemical staining except for Ctip2-staining, we used the same procedure. Sections were rinsed $3 \times 10 \mathrm{~min}$ in PBS-Tx followed by $60 \mathrm{~min}$ incubation in a blocking solution containing PBS-Tx with either $5 \%$ NGS or $3 \%$ Bovine serum albumin (BSA). 
Sections were incubated with the primary antibodies diluted in the blocking solution for $48 \mathrm{~h}$ at $4^{\circ} \mathrm{C}$, rinsed $3 \times 10 \mathrm{~min}$ in PBS-Tx, and incubated with secondary antibodies diluted in PBS-Tx overnight at room temperature. Finally, sections were rinsed $3 \times 10 \mathrm{~min}$ in PBS. For Ctip2-staining, sections were heated to $80^{\circ} \mathrm{C}$ for 15 minutes in $10 \mathrm{mM}$ sodium citrate ( $\mathrm{SC}, \mathrm{pH}$ 8.5). After cooling to room temperature, the sections were permeabilized by washing them 3 times in SC buffer containing $0.3 \%$ Triton X-100 (SC-Tx) and subsequently pre-incubated in a blocking solution containing SC-Tx and $3 \%$ BSA for one hour at room temperature. Next, sections were incubated with the primary antibodies diluted in the blocking solution for $48 \mathrm{~h}$ at $4^{\circ} \mathrm{C}$, rinsed $2 \times 15 \mathrm{~min}$ in SC-Tx and $1 \% \mathrm{BSA}$, and incubated with secondary antibodies diluted in SC-Tx and 1\%BSA for overnight at room temperature. Finally, sections were rinsed $3 \times 10$ min in PBS. After staining, sections were mounted on SuperfrostPlus microscope slides (Thermo Fisher Scientific) in Tris-gelatin solution ( $0.2 \%$ gelatin in Trisbuffer, $\mathrm{pH}$ 7.6), dried, and coverslipped with entellan in a toluene solution (Merck Chemicals, Darmstadt, Germany).

Coverslipped samples were imaged using an Axio ScanZ.1 fluorescent scanner, equipped with a 10× objective, Colibri. 2 LED light source, and a quadruple emission filter (PlanApochromat 10×, NA 0.45, excitation 488/546, emission 405/488/546/633, Carl Zeiss). To quantify the colocalization of GFP-, PCP4-, and Ctip2-immunolabeling, confocal images were acquired in sections taken at every $240 \mu \mathrm{m}$ throughout the entorhinal cortex, using a confocal microscope (Zeiss LSM 880 AxioImager Z2) with a 40× oil objective (Plan Apochromat 40x oil, NA 1.3, Carl Zeiss). The number of immunohistochemically labelled neurons was quantified in a fixed Z-level of the confocal images using Image $\mathbf{J}$ software (http://rsb.info.nih.gov/ij).

\section{Definition of EC and delineations of its layers}

The entorhinal cortex of rodents is typically defined as a ventral area of the cerebral cortex, delineated laterally by the rhinal sulcus and medially by the hippocampal formation. It shows a well-established typical laminar structure comprising two superficial and two deep cell layers, separated by a cell free layer, or lamina dissecans (Witter, 2011). Here we divide EC into LEC and MEC, mainly defined based on cytoarchitectural differences in LV, LIII, and LII. MEC has more columnar arrangement of LVb neurons compared to LEC. Layer III in MEC shows a sharp border with the lamina dissecans and has a very homogeneous appearance, whereas in LEC the border is les clear and the cellular organization is much more irregular. Finally, LEC can be identified by the presence of a superficial LII (LIIa) with densely packed neurons which tend to cluster into islands.

The borders of the superficial layers (I-III) and the thin acellular layer IV (lamina dissecans) were delineated as previously described (Witter, 2011). Layers Va and Vb were differentiated based on cell size, cell density, cell marker, and the projection patterns. LVb neurons are densely packed small cells that are PCP4-positive, whereas LVa is made up of sparsely distributed large cells which project to various cortical- and subcortical regions (Figure 1figure supplement 1) (Kitamura et al., 2017; Ohara et al., 2018; Sürmeli et al., 2015). The border between layer Vb and VI is more difficult to identify, since PCP4-staining also labels LVI neurons. We determined this border based on the cell density which decreases in LVI and the overall prominent orientation of neurons parallel to the pial surface (Witter, 2011). In case of MEC, the border can also be identified since the typical columnar organization of LVb stops upon entering LVI.

\section{Statistics}


Statistical analyses were performed using GraphPad Prism (GraphPad Software) or MATLAB (MathWorks). The details of tests used are described with the results. Differences between the groups were tested using paired and unpaired t-tests. Group comparisons were made using one-way ANOVA followed by Bonferroni post-hoc tests to control for multiple comparisons. All statistical tests were two-tailed, and thresholds for significance were placed at $* p<0.05$, $* * p<0.01$, and $* * * p<0.001$. All data are shown as mean \pm standard errors. No statistical methods were used to pre-determine sample size but the number of mice and cells for each experiment is similar with previous studies in the field (Doan et al., 2019; Nilssen et al., 2018). Mice were randomly selected from both sexes, and all experiments were successfully replicated in several samples. No blinding was used during data acquisition, but electrophysiological data analyses were performed blind to groups.

\section{Conflict of interest statement.}

The authors do not report a conflict of interest.

\section{Author contributions}

SO, MPW, MJN, and ESN conceived the study design. The experimental data was collected by SO and analysed by SO with help of MJN and ESN. SB produced the transgenic mouse line and RRN produced the AAV vectors, both under supervision of CGK. All authors contributed to the discussions that resulted in the current paper, which was written by SO and MPW, and edited by CGK. All authors approved the final version of the manuscript.

\section{Funding}

This work has been supported by the Kavli Foundation, the Centre of Excellence scheme Centre for Neural Computation and research grant \# 227769 of the Research Council of Norway, and the National Infrastructure scheme of the Research Council of Norway NORBRAIN \#197467. This work has also been supported by Grant-in-Aid for Scientific Research (KAKENHI, \#19K06917) from the Ministry of Education, Culture, Sports, Science and Technology (MEXT) of Japan.

\section{Acknowledgments}

We thank Grethe M. Olsen and Paulo Girao for help with histological preparations and microscopical imaging, and Paulo Girao and Yasutaka Honda for MATLAB programming. Bente Jacobsen and Thanh Doan contributed the tracing material for Figure 3-figure supplement 1 , and associated results described.

\section{References}

Alonso, A, Klink, R., 1993. Differential electroresponsiveness of stellate and pyramidal-like cells of medial entorhinal cortex layer II. J. Neurophysiol. 70(1), 128-143. 
Atasoy, D., Aponte, Y., Su, H.H., Sternson, S.M., 2008. A FLEX switch targets channelrhodopsin-2 to multiple cell types for imaging and long-range circuit mapping. J. Neurosci. 28(28), 7025-7030.

Beed, P., Bendels, M.H.K., Wiegand, H.F., Leibold, C., Johenning, F.W., Schmitz, D., 2010. Analysis of Excitatory Microcircuitry in the Medial Entorhinal Cortex Reveals CellType-Specific Differences. Neuron 68(6), 1059-1066.

Beed, P., de Filippo, R., Holman, C., Johenning, F.W., Leibold, C., Caputi, A., Monyer, H., Schmitz, D., 2020. Layer 3 Pyramidal Cells in the Medial Entorhinal Cortex Orchestrate Up-Down States and Entrain the Deep Layers Differentially. Cell Rep. 33(10), 108470.

Bellmund, J.L., Deuker, L., Doeller, C.F., 2019. Mapping sequence structure in the human lateral entorhinal cortex. Elife 8.

Blankvoort, S., Witter, M.P., Noonan, J., Cotney, J., Kentros, C., 2018. Marked Diversity of Unique Cortical Enhancers Enables Neuron-Specific Tools by Enhancer-Driven Gene Expression. Curr. Biol. 28(13), 2103-2114.e5.

Bonnevie, T., Dunn, B., Fyhn, M., Hafting, T., Derdikman, D., Kubie, J.L., Roudi, Y., Moser, E.I., Moser, M.B., 2013. Grid cells require excitatory drive from the hippocampus. Nat. Neurosci. 16(3), 309-317.

Buzsáki, G., 1996. The hippocampo-neocortical dialogue. Cereb. Cortex 6, 81-92.

Canto, C.B., Koganezawa, N., Beed, P., Moser, E.I., Witter, M.P., 2012. All Layers of Medial Entorhinal Cortex Receive Presubicular and Parasubicular Inputs. J. Neurosci. 32(49), 17620-17631.

Canto, C.B., Witter, M.P., 2012a. Cellular properties of principal neurons in the rat entorhinal cortex. II. The medial entorhinal cortex. Hippocampus 22(6), 1277-1299.

Canto, C.B., Witter, M.P., 2012b. Cellular properties of principal neurons in the rat entorhinal cortex. I. The lateral entorhinal cortex. Hippocampus 22(6), 1256-1276.

Chrobak, J.J., Amaral, D.G., 2007. Entorhinal cortex of the monkey: VII. Intrinsic connections. J. Comp. Neurol. 500(4), 612-633.

Couey, J.J., Witoelar, A., Zhang, S.J., Zheng, K., Ye, J., Dunn, B., Czajkowski, R., Moser, M.B., Moser, E.I., Roudi, Y., Witter, M.P., 2013. Recurrent inhibitory circuitry as a mechanism for grid formation. Nat. Neurosci. 16(3), 318-24.

Deshmukh, S.S., Knierim, J.J., 2011. Representation of Non-Spatial and Spatial Information in the Lateral Entorhinal Cortex. Front. Behav. Neurosci. 5(69).

Doan, T.P., Lagartos-Donate, M.J., Nilssen, E.S., Ohara, S., Witter, M.P., 2019. Convergent Projections from Perirhinal and Postrhinal Cortices Suggest a Multisensory Nature of Lateral, but Not Medial, Entorhinal Cortex. Cell Rep. 29(3), 617-627.e7.

Edelman, G.M., 1989. The Remembered Present : A Biological Theory of Consciousness. Basic Books.

Egorov, A. V., Hamam, B.N., Fransén, E., Hasselmo, M.E., Alonso, A.A., 2002. Graded persistent activity in entorhinal cortex neurons. Nature 420(6912), 173-178. 
Egorov, A. V., Heinemann, U., Müller, W., 2002. Differential excitability and voltagedependent $\mathrm{Ca} 2+$ signalling in two types of medial entorhinal cortex layer $\mathrm{V}$ neurons. Eur. J. Neurosci. 16(7), 1305-1312.

Eichenbaum, H., Sauvage, M., Fortin, N., Komorowski, R., Lipton, P., 2012. Towards a functional organization of episodic memory in the medial temporal lobe. Neurosci. Biobehav. Rev. 36(7), 1597-1608.

Frankland, P.W., Bontempi, B., 2005. The organization of recent and remote memories. Nat. Rev. Neurosci. 6(2), 119-130.

Fransén, E., Tahvildari, B., Egorov, A. V., Hasselmo, M.E., Alonso, A.A., 2006. Mechanism of graded persistent cellular activity of entorhinal cortex layer V neurons. Neuron 49(5), $735-746$.

Fuchs, E.C., Neitz, A., Pinna, R., Melzer, S., Caputi, A., Monyer, H., 2016. Local and Distant Input Controlling Excitation in Layer II of the Medial Entorhinal Cortex. Neuron 89(1), 194-208.

Griesbeck, O., Baird, G.S., Campbell, R.E., Zacharias, D.A., Tsien, R.Y., 2001. Reducing the environmental sensitivity of yellow fluorescent protein. Mechanism and applications. J. Biol. Chem. 276(31), 29188-94.

Hafting, T., Fyhn, M., Molden, S., Moser, M.B., Moser, E.I., 2005. Microstructure of a spatial map in the entorhinal cortex. Nature 436(7052), 801-806.

Hamam, B.N., Amaral, D.G., Alonso, A.A., 2002. Morphological and electrophysiological characteristics of layer $\mathrm{V}$ neurons of the rat lateral entorhinal cortex. J. Comp. Neurol. 451(1), 45-61.

Hamam, B.N., Kennedy, T.E., Alonso, A., Amaral, D.G., 2000. Morphological and electrophysiological characteristics of layer $\mathrm{V}$ neurons of the rat medial entorhinal cortex. J. Comp. Neurol. 418(4), 457-472.

Iijima, T., Witter, M.P., Ichikawa, M., Tominaga, T., Kajiwara, R., Matsumoto, G., 1996. Entorhinal-hippocampal interactions revealed by real-time imaging. Science 272(5265), 1176-1179.

Kitamura, T., Ogawa, S.K., Roy, D.S., Okuyama, T., Morrissey, M.D., Smith, L.M., Redondo, R.L., Tonegawa, S., 2017. Engrams and circuits crucial for systems consolidation of a memory. Science 356(6333), 73-78.

Kloosterman, F., van Haeften, T., Lopes da Silva, F.H., 2004. Two reentrant pathways in the hippocampal-entorhinal system. Hippocampus 14(8), 1026-1039.

Köhler, C., 1986. Intrinsic connections of the retrohippocampal region in the rat brain. II. The medial entorhinal area. J. Comp. Neurol. 246(2), 149-169.

Köhler, C., 1985. Intrinsic projections of the retrohippocampal region in the rat brain. I. The subicular complex. J. Comp. Neurol. 236(4), 504-22.

Kosel, K.C., Van Hoesen, G.W., Rosene, D.L., 1982. Non-hippocampal cortical projections from the entorhinal cortex in the rat and rhesus monkey. Brain Res. 244(2), 201-213. 
Leitner, F.C., Melzer, S., Lütcke, H., Pinna, R., Seeburg, P.H., Helmchen, F., Monyer, H., 2016. Spatially segregated feedforward and feedback neurons support differential odor processing in the lateral entorhinal cortex. Nat. Neurosci. 19(7), 935-944.

Lin, J.Y., Lin, M.Z., Steinbach, P., Tsien, R.Y., 2009. Characterization of engineered channelrhodopsin variants with improved properties and kinetics. Biophys. J. 96(5), 1803-14.

Luskin, M.B., Price, J.L., 1983. The topographic organization of associational fibers of the olfactory system in the rat, including centrifugal fibers to the olfactory bulb. J. Comp. Neurol. 216, 264-291.

Lux, V., Atucha, E., Kitsukawa, T., Sauvage, M.M., 2016. Imaging a memory trace over half a life-time in the medial temporal lobe reveals a time-limited role of CA3 neurons in retrieval. Elife 5.

Madisen, L., Zwingman, T.A., Sunkin, S.M., Oh, S.W., Zariwala, H.A., Gu, H., Ng, L.L., Palmiter, R.D., Hawrylycz, M.J., Jones, A.R., Lein, E.S., Zeng, H., 2010. A robust and high-throughput Cre reporting and characterization system for the whole mouse brain. Nat. Neurosci. 13(1), 133-140.

Montchal, M.E., Reagh, Z.M., Yassa, M.A., 2019. Precise temporal memories are supported by the lateral entorhinal cortex in humans. Nat. Neurosci. 22(2), 284-288.

Moser, E.I., Moser, M.B., McNaughton, B.L., 2017. Spatial representation in the hippocampal formation: A history. Nat. Neurosci. 20(11), 1448-1464.

Nadel, L., Moscovitch, M., 1997. Memory consolidation, retrograde amnesia and the hippocampal complex. Curr. Opin. Neurobiol. 7(2), 217-227.

Nair, R.R., Blankvoort, S., Lagartos, M.J., Kentros, C., 2020. Enhancer-Driven Gene Expression (EDGE) Enables the Generation of Viral Vectors Specific to Neuronal Subtypes. iScience 23(3), 100888.

Nilssen, E.S., Jacobsen, B., Fjeld, G., Nair, R.R., Blankvoort, S., Kentros, C., Witter, M.P., 2018. Inhibitory connectivity dominates the fan cell network in layer II of lateral entorhinal cortex. J. Neurosci. 38(45), 9712-9727.

Nilssen, E.S., 2019. Synaptic connectivity of principal cells in layer II of the lateral entorhinal cortex. PhD Thesis NTNU. ISSN 1503-8181.

Ohara, S., Gianatti, M., Itou, K., Berndtsson, C.H., Doan, T.P., Kitanishi, T., Mizuseki, K., Iijima, T., Tsutsui, K.I., Witter, M.P., 2019. Entorhinal Layer II Calbindin-Expressing Neurons Originate Widespread Telencephalic and Intrinsic Projections. Front. Syst. Neurosci. 13(54).

Ohara, S., Onodera, M., Simonsen, Ø.W., Yoshino, R., Hioki, H., Iijima, T., Tsutsui, K.I., Witter, M.P., 2018. Intrinsic Projections of Layer Vb Neurons to Layers Va, III, and II in the Lateral and Medial Entorhinal Cortex of the Rat. Cell Rep. 24(1), 107-116.

Ramsden, H.L., Sürmeli, G., McDonagh, S.G., Nolan, M.F., 2015. Laminar and Dorsoventral Molecular Organization of the Medial Entorhinal Cortex Revealed by Large-scale Anatomical Analysis of Gene Expression. PLoS Comput. Biol. 11(1), 1-38.

Rosene, D.L., Van Hoesen, G.W., 1977. Hippocampal efferents reach widespread areas of cerebral cortex and amygdala in the rhesus monkey. Science 198(4314), 315-317. 
Rozov, A., Rannap, M., Lorenz, F., Nasretdinov, A., Draguhn, A., Egorov, A. V., 2020. Processing of Hippocampal Network Activity in the Receiver Network of the Medial Entorhinal Cortex Layer V. J. Neurosci. 40(44), 8413-8425.

Schmitz, D., Gloveli, T., Behr, J., Dugladze, T., Heinemann, U., 1998. Subthreshold membrane potential oscillations in neurons of deep layers of the entorhinal cortex. Neuroscience 85(4), 999-1004.

Squire, L.R., Alvarez, P., 1995. Retrograde amnesia and memory consolidation: a neurobiological perspective. Curr. Opin. Neurobiol. 5(2), 169-177.

Steffenach, H.A., Witter, M.P., Moser, M.B., Moser, E.I., 2005. Spatial memory in the rat requires the dorsolateral band of the entorhinal cortex. Neuron 45(2), 301-313.

Stensola, H., Stensola, T., Solstad, T., Froland, K., Moser, M.-B., Moser, E.I., 2012. The entorhinal grid map is discretized. Nature 492(7427), 72-78.

Steward, O., Scoville, S.A., 1976. Cells of origin of entorhinal cortical afferents to the hippocampus and fascia dentata of the rat. J. Comp. Neurol. 169(3), 347-70.

Suh, J., Rivest, A.J., Nakashiba, T., Tominaga, T., Tonegawa, S., 2011. Entorhinal cortex layer III input to the hippocampus is crucial for temporal association memory. Science 334(6061), 1415-1420.

Sürmeli, G., Marcu, D.C., McClure, C., Garden, D.L.F., Pastoll, H., Nolan, M.F., 2015. Molecularly Defined Circuitry Reveals Input-Output Segregation in Deep Layers of the Medial Entorhinal Cortex. Neuron 88(5), 1040-1053.

Tanaka, D., Nakaya, Y., Yanagawa, Y., Obata, K., Murakami, F., 2003. Multimodal tangential migration of neocortical GABAergic neurons independent of GPI-anchored proteins. Development 130(23), 5803-13.

Tang, Q., Burgalossi, A., Ebbesen, C.L., Ray, S., Naumann, R., Schmidt, H., Spicher, D., Brecht, M., 2014. Pyramidal and stellate cell specificity of grid and border representations in layer 2 of medial entorhinal cortex. Neuron 84(6), 1191-1197.

Tsao, A., Moser, M.B., Moser, E.I., 2013. Traces of experience in the lateral entorhinal cortex. Curr. Biol. 23(5), 399-405.

Tsao, A., Sugar, J., Lu, L., Wang, C., Knierim, J.J., Moser, M.-B., Moser, E.I., 2018. Integrating time from experience in the lateral entorhinal cortex. Nature 561(7721), 5762.

Varga, C., Lee, S.Y., Soltesz, I., 2010. Target-selective GABAergic control of entorhinal cortex output. Nat. Neurosci. 13(7), 822-4.

Winterer, J., Maier, N., Wozny, C., Beed, P., Breustedt, J., Evangelista, R., Peng, Y., Albis, T.D., Kempter, R., Schmitz, D., 2017. Excitatory microcircuits within superficial layers of the medial entorhinal cortex. Cell Rep. 1247, 1110-1116.

Witter, M.P. (2011). The hippocampus. In The Mouse Nervous System, First Edition, Paxinos G., Puilles L., and Watson C., eds. (Academic), pp. 112-139

Wozny, C., Beed, P., Nitzan, N., Pössnecker, Y., Rost, B.R., Schmitz, D., 2018. VGLUT2 functions as a differential marker for hippocampal output neurons. Front. Cell. Neurosci. $12: 337$. 
$946 \mathrm{Xu}, \mathrm{W} ., \mathrm{Wilson}, \mathrm{D} . \mathrm{A} ., 2$ 2012. Odor-evoked activity in the mouse lateral entorhinal cortex. Neuroscience 223, 12-20. 

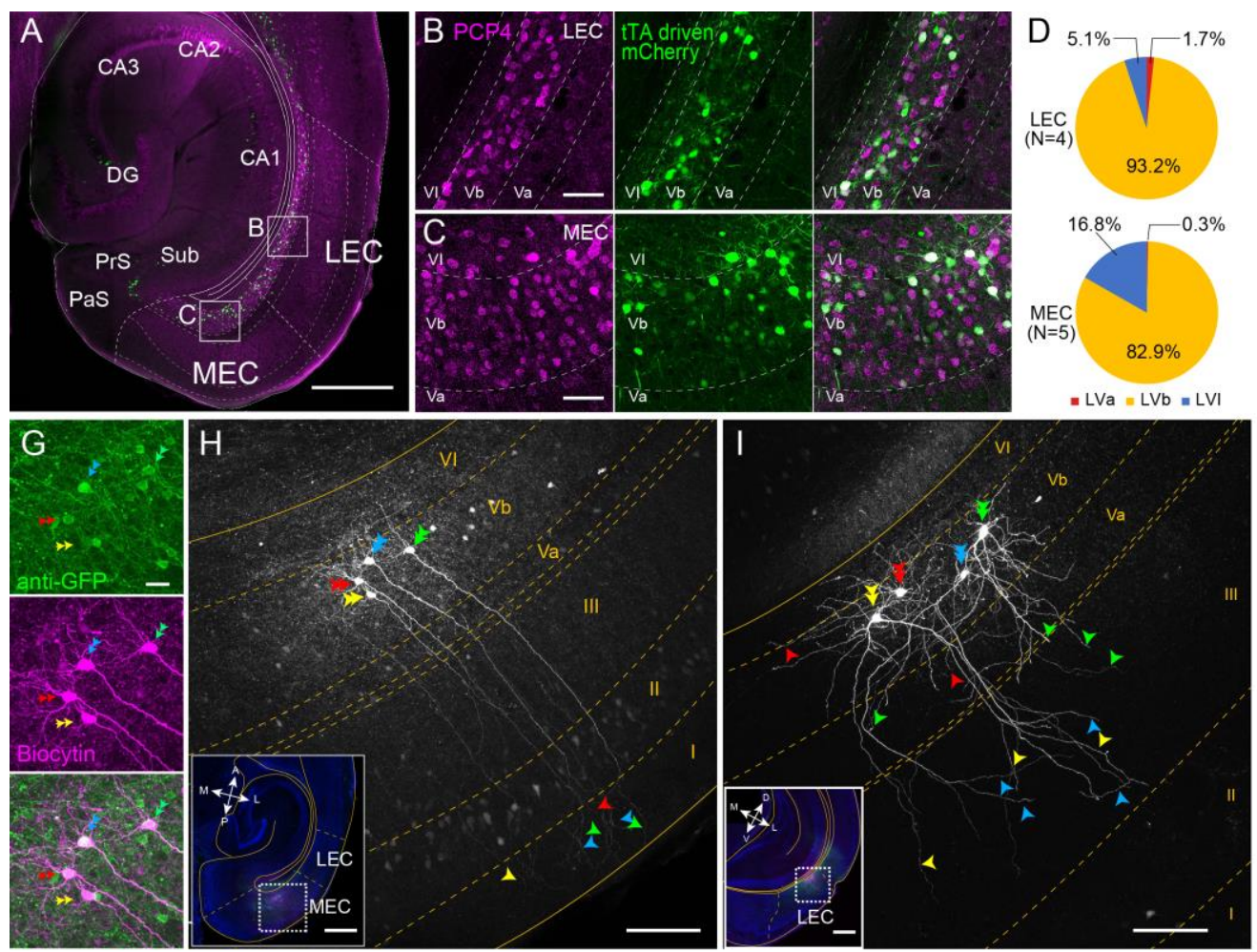
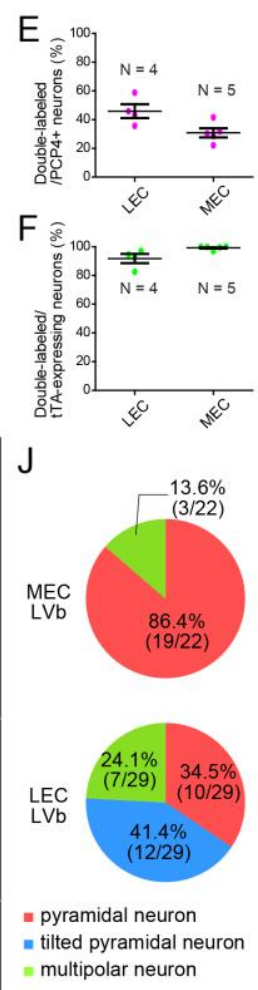

Figure 1. LEC and MEC LVb neurons show distinct morphological features.

A-C, Expression of tTA in the EDGE mouse line (MEC-13-53D), which is visualized with mCherry (green) by crossing to a tTA-dependent mCherry line. A horizontal section was immunostained with an anti-PCP4 antibody (magenta) to label entorhinal LVb neurons. Images of LEC (B) and MEC (C) correspond with the boxed areas in (A) and show from left to right PCP4 expression, mCherry expression and a merged image. D, Percentage of tTAexpressing neurons among layers in LEC and MEC. E, Percentage of tTA-expressing neurons among the total PCP4-positive neurons in LEC and MEC. F, Percentage of PCP4-positive neurons among the total tTA-expressing neurons in LEC and MEC. Error bars: mean \pm standard errors. The tTA-expressing neurons mainly distributed in LVb of EC and colocalized with PCP4. G-I, Morphology of LVb neurons targeted in MEC-13-53D in MEC (G-H) and LEC (I). tTA-expressing LVb neurons were first labelled with GFP (green) by injecting AAV2/1-TRE-Tight-EGFP in MEC-13-53D, and then intracellularly filled with biocytin (magenta, $\mathrm{G})$. Images of MEC $(\mathrm{H})$ and LEC (I) show biocytin labeling which correspond with the boxed area in each inset. The four neurons shown in $(G)$ correspond to the neurons in $(\mathrm{H})$. Double arrowheads show the cell bodies and the single arrowheads show their dendrites, and different neurons are marked in different colors (green, blue, red, and yellow). The distribution of apical dendrites largely differs between MEC-LVb and LECLVb neurons. J, Proportion of morphologically identified cell types of LVb neurons in LEC and MEC. These data were obtained in 10 animals and 22 slices. Scale bars represent $500 \mu \mathrm{m}$ for (A) and inset of (H) and (I), $100 \mu \mathrm{m}$ for (H) and (I), $50 \mu \mathrm{m}$ for (B) and (C), and $20 \mu \mathrm{m}$ for (G). Figure 1-source data 1. See also Figure 1-figure supplement 1, Figure 1-figure supplement 2, Figure 1-figure supplement 3, and Figure 1-figure supplement 4. 

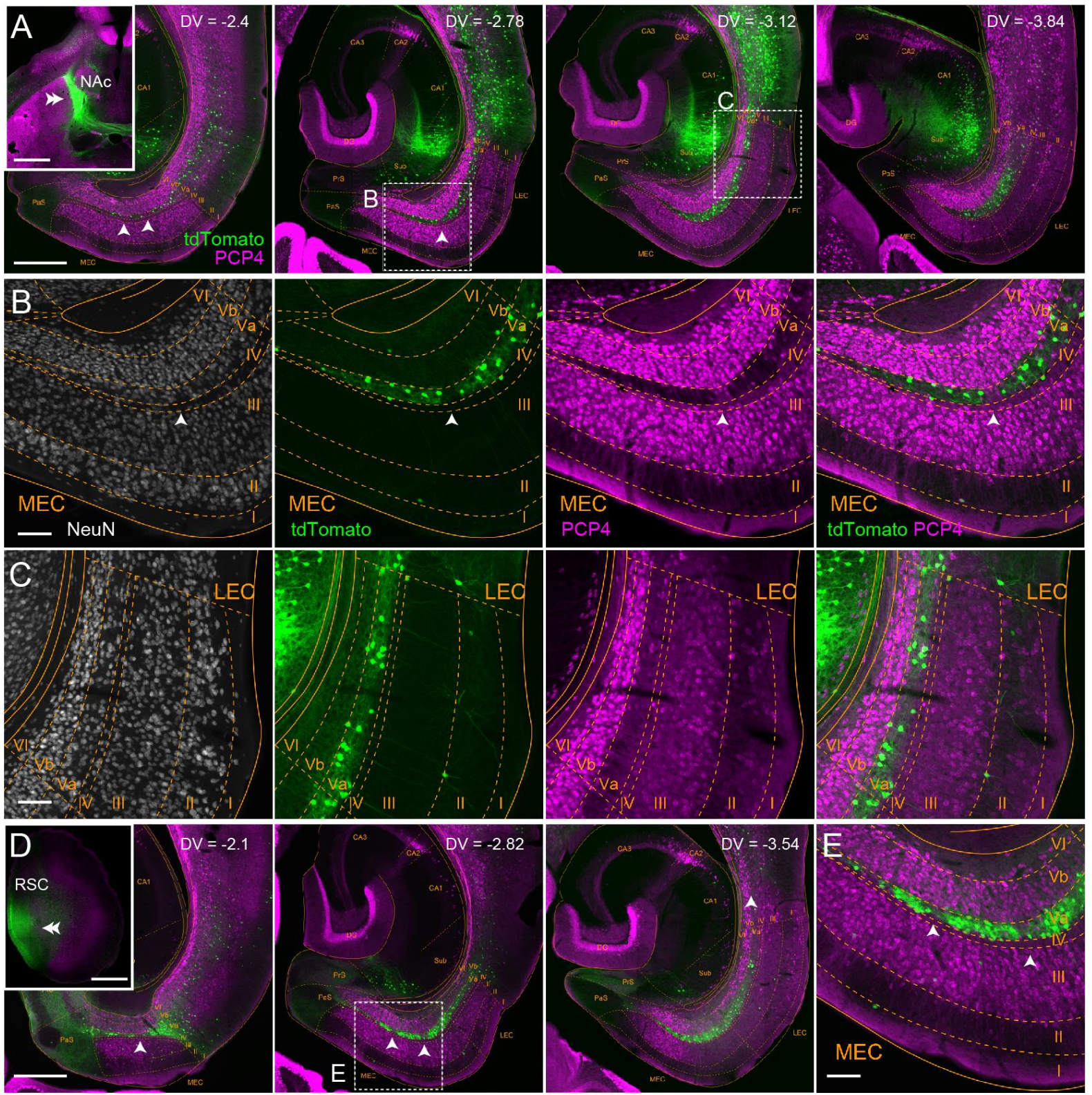

Figure 1-figure supplement 1. Laminar organization of LEC and MEC.

A, Horizontal sections showing PCP4-immunolabeling (magenta) and retrograde-labeling with tdTomato (green) at different dorsoventral levels, following injection of Cre-expressing retrograde AAV (AAVrg-pmSyn1-EBFP-cre) into nucleus accumbens (NAc) of Rosa26TdTomatoAi9 mice. The injection site is shown by a double-arrowhead in the inset. Note that PCP4-positive neurons are localized in LVb, whereas NAc-projecting neurons distribute in LVa of both MEC and LEC. The thickness of EC LVa/LVb varies along the dorsoventral- and mediolateral-axis, and between LEC and MEC. LVa is especially thin in the medial and dorsal portion of MEC, whereas LVb is relatively thick. LVa gets thicker in lateral and ventral portions, and in the LEC the thickness of LVa and LVb becomes similar. B-C, Distribution of NeuN-, tdTomato-, and PCP4-positive neurons in MEC (B) and LEC (C) corresponding with the boxed area in (A). White arrowheads show the gaps between the tdTomato-positive retrogradely labeled MEC-LVa neurons which are also shown in (A) and (D). Note that in MEC these gaps do contain PCP4-positive neurons, as well as PCP4positive neuropil likely representing apical dendrites of $\mathrm{LVb}$ neurons. This patchy pattern of alternating somata of neurons and neuropil was never observed in LVa of LEC. D-E, 
993 Distribution of PCP4-positive neurons and retrogradely-labeled neurons in MEC following 994 AAVrg-pmSyn1-EBFP-cre injection into retrosplenial cortex (RSC) of Rosa26TdTomatoAi9 995 mice. Double arrowhead in the inset indicates the injection site. Scale bars represent $1000 \mu \mathrm{m}$ 996 for insets in (A) and (D), $500 \mu \mathrm{m}$ for EC images in (A) and (D), $100 \mu \mathrm{m}$ for (B), (C), and (E). 

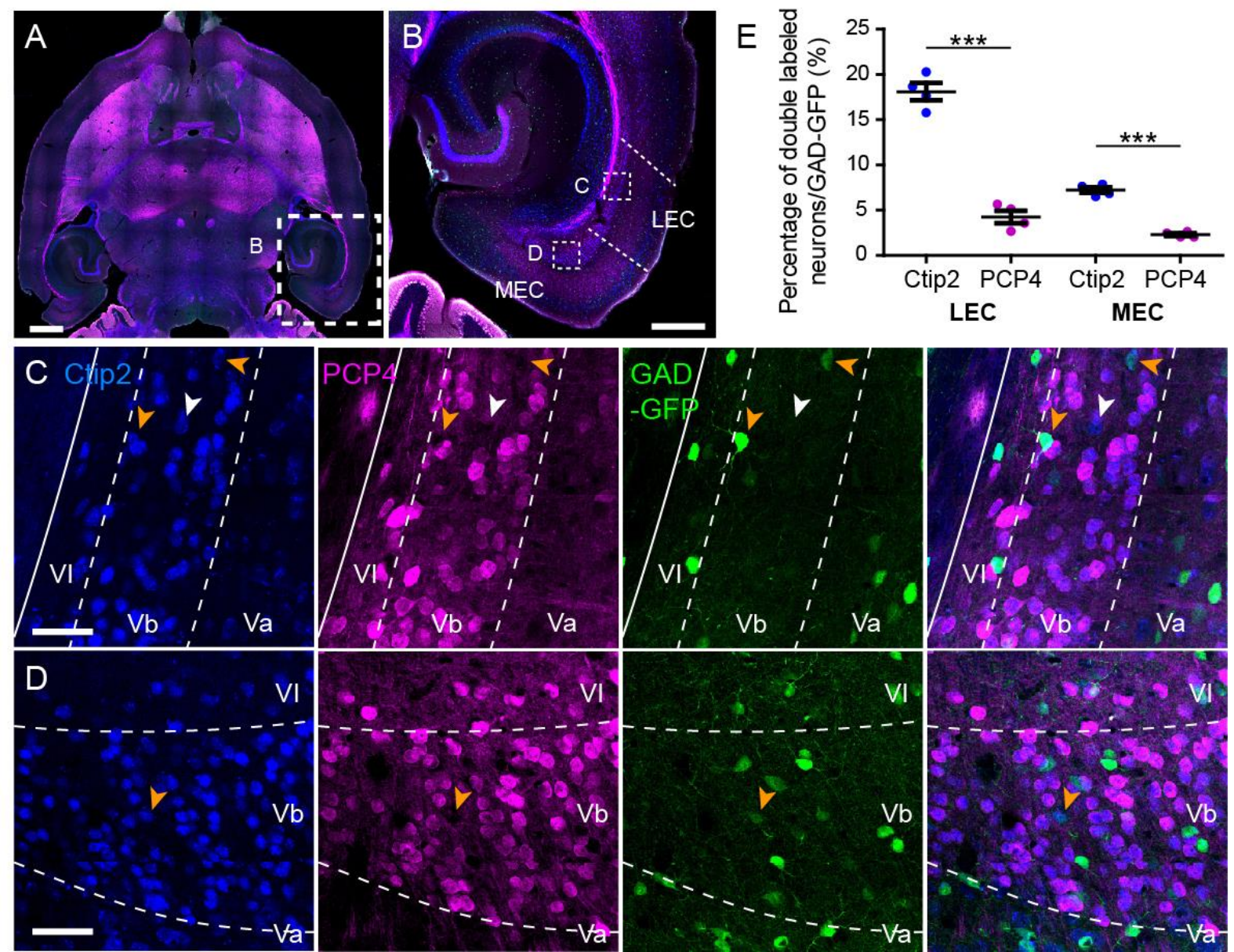

Figure 1-figure supplement 2. PCP4 but not Ctip2 is expressed mainly in excitatory LVb neurons in both LEC and MEC.

A-B, Horizontal section showing Ctip2- (blue) and PCP4-immunolabeling (magenta). C-D, Distribution of Ctip2- (blue), PCP4- (magenta), and GAD67-GFP-positive neurons (green) in LEC (C) and MEC (D) corresponding with the boxed areas in (B). White arrowheads point to Ctip2+/PCP4-/GAD67- neurons, while yellow arrowheads point to Ctip2+/PCP4-/GAD67+ neurons. E, In both LEC and MEC, the percentage of GAD67-GFP positive neurons doublelabeled for Ctip2 (Ctip2+/GAD67+; blue) was significantly higher than the percentage double-labeled for PCP4 (PCP4+/GAD67+; magenta) (Error bars: mean \pm standard errors, $\mathrm{N}$ $=5$, two-tailed paired t-test for LEC Ctip2 vs PCP4: $\mathrm{t} 3=15.34, * * * \mathrm{p}=0.0006$, MEC Ctip2 vs PCP4: $\mathrm{t} 3=13.74, * * * \mathrm{p}=0.0008)$. Scale bars represent $1000 \mu \mathrm{m}$ for insets in (A), $500 \mu \mathrm{m}$ for (B), $50 \mu \mathrm{m}$ for (C) and (D). Figure 1-figure supplement 2-source data 1. 

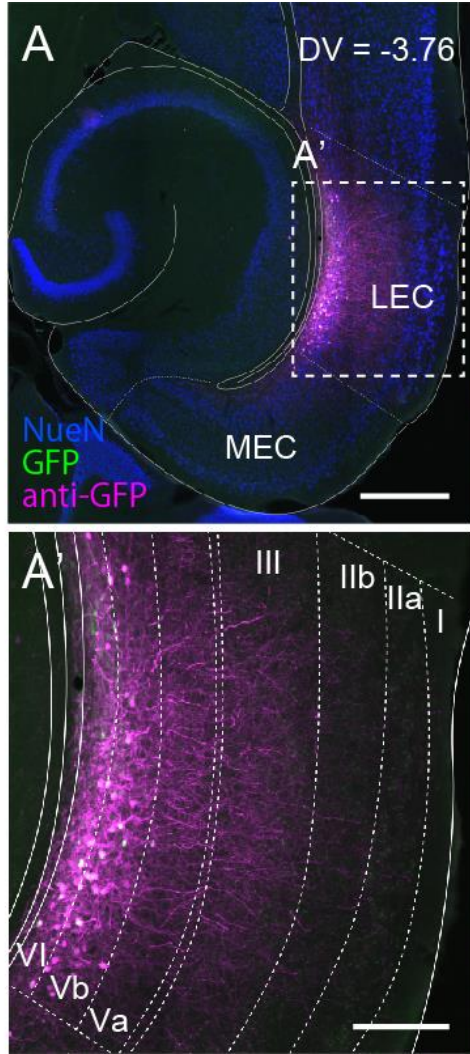
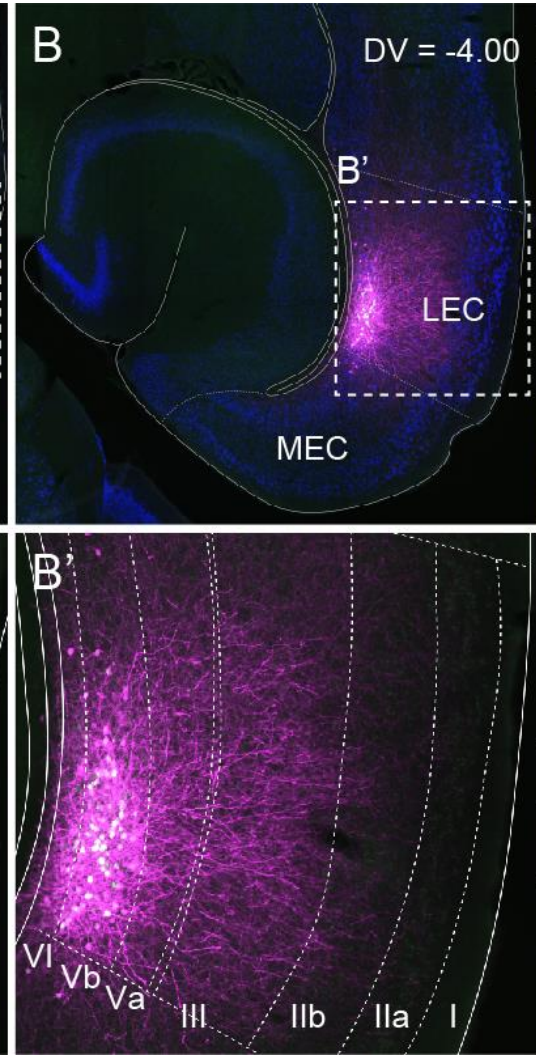

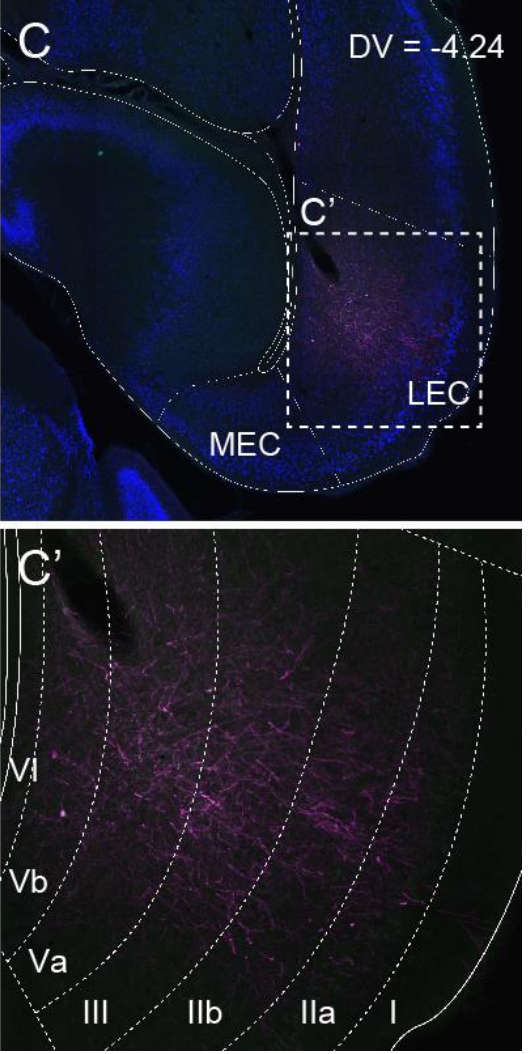

Figure 1-figure supplement 3. Dendrites of LEC-LVb neurons do not reach layer IIa and $\mathbf{I}$.

Horizontal sections showing the distribution of GFP-labeled LEC-LVb neurons at different dorsoventral levels, following injection of AAV2/1-TRE-Tight-GFP into the deep layers of LEC. Neurites of labeled LEC-LVb neurons are visualized with anti-GFP staining shown in magenta. Cell bodies of these infected cells can be seen in white resulting from the overlap of GFP (green) and anti-GFP labelling (magenta). Scale bars represent $500 \mu \mathrm{m}$ for (A) (also apply to B and C), $200 \mu \mathrm{m}$ for (A') (also apply to B' and C'). 

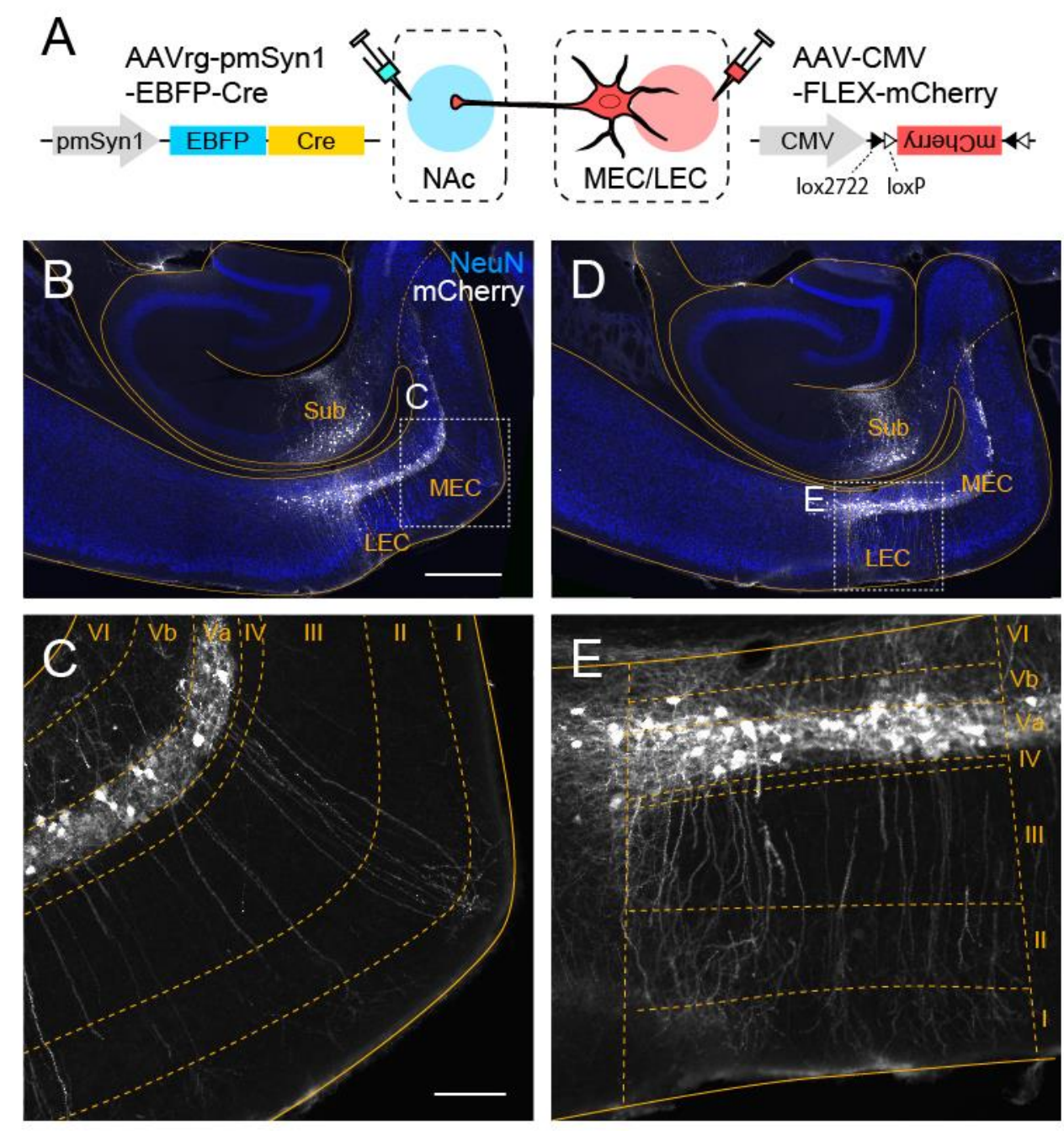

Figure 1-figure supplement 4. MEC- and LEC-LVa neurons share similar morphological features.

A, LVa neurons were labeled in C57BL/6N mice, by injecting Cre-expressing retrograde AAV (AAVrg-pmSyn1-EBFP-cre) in NAc while injecting AAV-CMV-FLEX-mCherry in EC. B-E, Horizontal sections showing labeled LVa neurons in MEC (C) and LEC (E), corresponding with the boxed area in (B) and (D), respectively. In both regions, the apical dendrites of LVa neurons reach layer I. Labeled neurons in the hippocampus (B, D) are NAcprojecting subicular neurons which were also infected due to the spread of AAV-CMVFLEX-mCherry from the injection site. Scale bars represent $500 \mu \mathrm{m}$ for (B) (also apply to D), $100 \mu \mathrm{m}$ for (C) (also apply to E). 

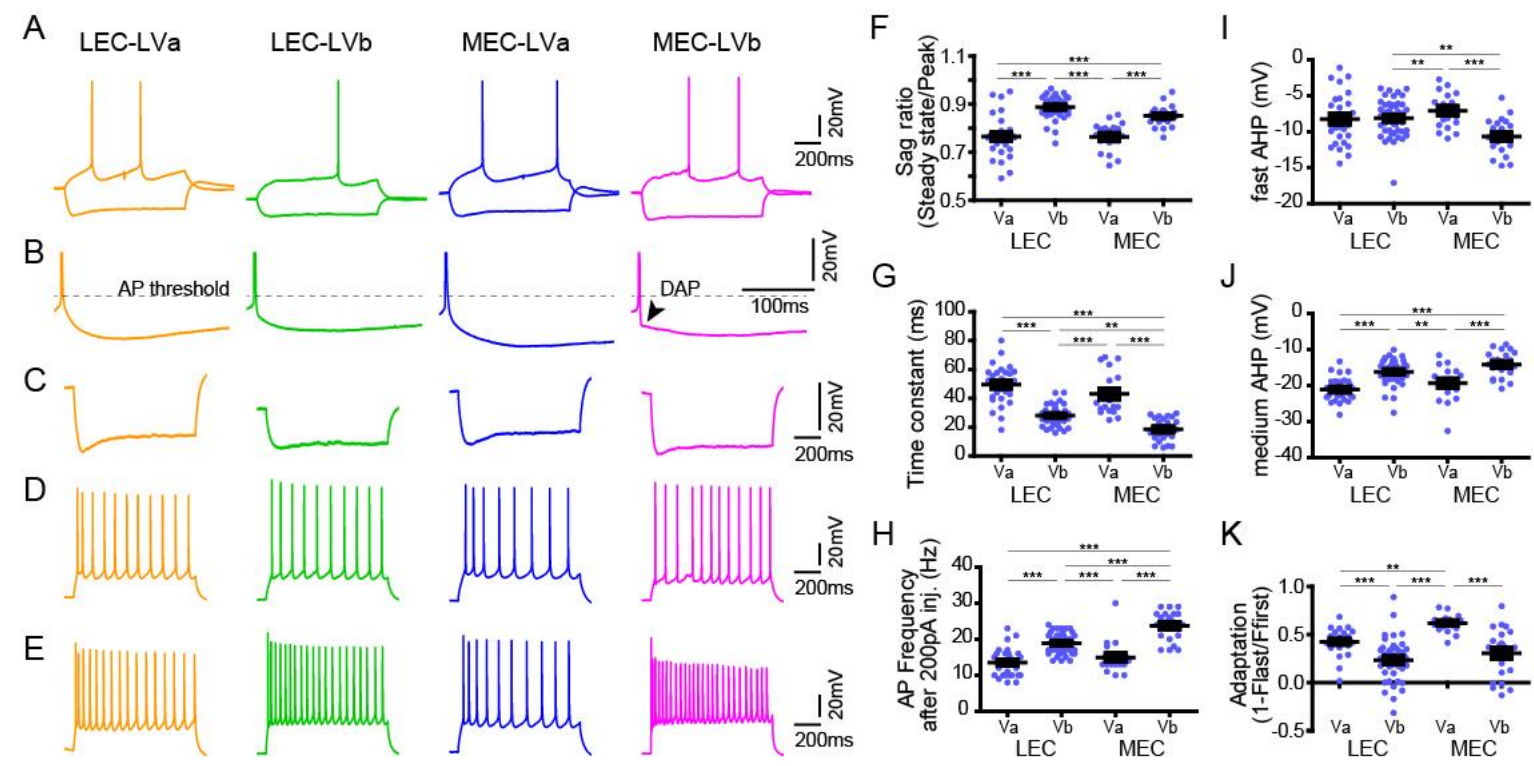

L

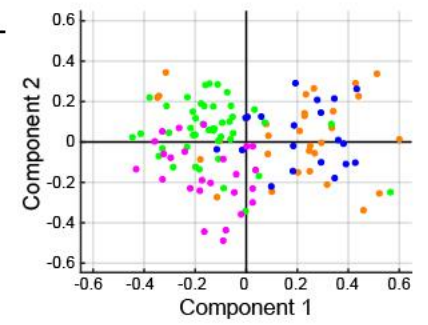

- LEC-LVa • LEC-LVb • MEC-LVa • MEC-LVb
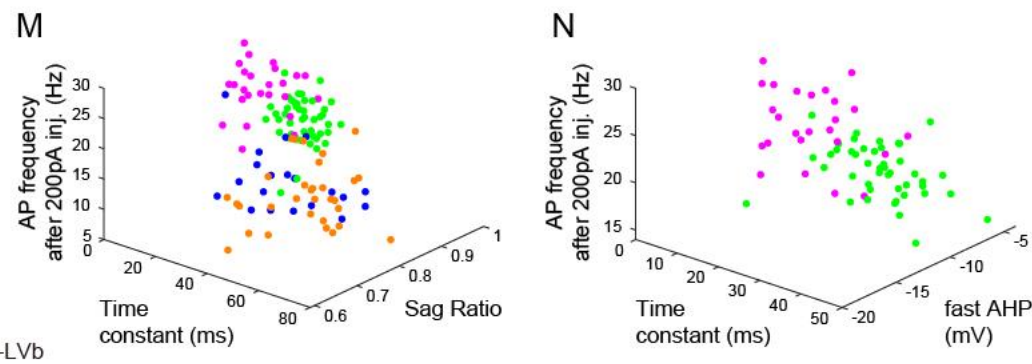

Figure 2. Electrophysiological properties distinguish $\mathrm{LVa} / \mathrm{LVb}$ neurons in both $\mathrm{LEC}$ and MEC.

A, Representative voltage responses to hyperpolarizing and depolarizing current injection of LEC-LVa (orange), LEC-LVb (green), MEC-LVa (blue), and MEC-LVb (magenta) neurons. $\mathbf{B}$, Voltage responses at rheobase current injections showing AHP wave form and DAP. C, Voltage responses to hyperpolarizing current injection with peaks at $-90 \pm 5 \mathrm{mV}$ showing Sag. D, Voltage responses to depolarizing current injection with $10 \pm 1$ APs showing adaptation. E, Voltage responses to $+200 \mathrm{pA}$ of 1-s-long current injection showing maximal AP number. F$\mathbf{K}$, Differences of sag ratio (F, one-way ANOVA, $F_{3,117}=36.88$, *** $p<0.0001$, Bonferroni's multiple comparison test, $* * * p<0.001)$, time constant $\left(\mathrm{G}\right.$, one-way ANOVA, $F_{3,117}=53.39$, $* * * p<0.0001$, Bonferroni's multiple comparison test, $* * p<0.01, * * * p<0.001$ ), AP frequency after $200 \mathrm{pA}$ injection $\left(\mathrm{H}\right.$, one-way ANOVA, $F_{3,117}=44.37$, $* * * p<0.0001$, Bonferroni's multiple comparison test, ${ }^{* * *} p<0.001$ ), fast AHP (I, one-way ANOVA, $\mathrm{F} 3,117=7.536, * * * \mathrm{p}=0.0001$, Bonferroni's multiple comparison test, $* * \mathrm{p}<0.01,{ }^{* * *} \mathrm{p}<$ $0.001)$, medium AHP (J, one-way ANOVA, $F_{3,117}=21.99$, $* * * p<0.0001$, Bonferroni's multiple comparison test, $* * p<0.01, * * * p<0.001)$, and adaptation $(\mathrm{K}$, one-way ANOVA, $F_{3,117}=21.6, * * * p<0.0001$, Bonferroni's multiple comparison test, $* * p<0.01, * * * p<$ $0.001)$ between LEC-LVa (N=31), LEC-LVb (N=45), MEC-LVa (N=20), and MEC-LVb $(\mathrm{N}=25)$ neurons (Error bars: mean \pm standard errors). L, Principal component analysis based on the twelve electrophysiological parameters shown in Figure 2-figure supplement 1 show a separation between LVa and LVb neurons as well as a moderate separation between LEC$\mathrm{LVb}$ and MEC-LVb neurons. Data representing 121 neurons from 27 animals (also holds for MEC-LVb (magenta) neurons using sag ratio, AP frequency at 200 pA injection, and time 
constant as distinction criteria. N, Separation of LEC-LVb (green) and MEC-LVb (magenta) neurons using fast AHP, AP frequency at $200 \mathrm{pA}$ injection, and time constant as distinction criteria. Figure 2-source data 1. See also Figure 2-figure supplement 1 and Figure 2- 


\begin{tabular}{|c|c|c|c|c|}
\hline Parameter & $\begin{array}{l}\text { LEC Va neurons } \\
\quad(\mathrm{N}=31)\end{array}$ & $\begin{array}{l}\text { LEC Vb neurons } \\
(\mathrm{N}=45)\end{array}$ & $\begin{array}{l}\text { MEC Va neurons } \\
(\mathrm{N}=20)\end{array}$ & $\begin{array}{c}\text { MEC Vb neurons } \\
(\mathrm{N}=25)\end{array}$ \\
\hline $\mathrm{V}_{\text {rest }}(\mathrm{mV})$ & $-63.1 \pm 0.6$ & $-64.8 \pm 0.5$ & $-64.6 \pm 1.0$ & $-65.0 \pm 0.6$ \\
\hline $\mathrm{R}_{\text {in }}(\mathrm{M} \Omega)$ & $294.4 \pm 18.7$ & $269.0 \pm 11.5$ & $338.4 \pm 26.8$ & $352.9 \pm 27.9$ \\
\hline Time constant (msec) & $49.6 \pm 2.3$ & $28.1 \pm 0.9$ & $43.1 \pm 3.1$ & $18.5 \pm 1.5$ \\
\hline Sag ratio (Steady state/Peak) & $0.77 \pm 0.01$ & $0.89 \pm 0.01$ & $0.76 \pm 0.01$ & $0.85 \pm 0.01$ \\
\hline $\mathrm{AP}_{\text {thre }}(\mathrm{mV})$ & $-34.8 \pm 0.5$ & $-36.1 \pm 0.5$ & $-34.3 \pm 0.7$ & $-34.7 \pm 0.6$ \\
\hline $\mathrm{AP}_{\text {peak }}(\mathrm{mV})$ & $86.8 \pm 1.5$ & $91.0 \pm 0.8$ & $83.8 \pm 1.2$ & $88.6 \pm 1.1$ \\
\hline $\mathrm{AP}_{\mathrm{HW}}(\mathrm{ms})$ & $1.20 \pm 0.04$ & $1.35 \pm 0.02$ & $1.26 \pm 0.05$ & $1.19 \pm 0.02$ \\
\hline$A P_{\text {rise }}(\mathrm{mV} / \mathrm{ms})$ & $367.1 \pm 15.3$ & $397.1 \pm 9.7$ & $341.8 \pm 15.4$ & $382.0 \pm 14.2$ \\
\hline Fast AHP (mV) & $-8.24 \pm 0.60$ & $-8.10 \pm 0.38$ & $-7.08 \pm 0.51$ & $-10.66 \pm 0.44$ \\
\hline Medium AHP (mV) & $-21.1 \pm 0.6$ & $-16.2 \pm 0.5$ & $-19.4 \pm 10.2$ & $-14.2 \pm 0.7$ \\
\hline AP frequency after $200 \mathrm{pA}$ inj. $(\mathrm{Hz})$ & $13.5 \pm 0.7$ & $18.9 \pm 0.4$ & $15.0 \pm 0.9$ & $23.8 \pm 0.7$ \\
\hline Adaption $\left(1-F_{\text {last }} / F_{\text {first }}\right)$ & $0.42 \pm 0.02$ & $0.23 \pm 0.03$ & $0.62 \pm 0.02$ & $0.31 \pm 0.05$ \\
\hline
\end{tabular}

1066 Figure 2-figure supplement 1. Table showing the electrophysiological properties of 1067 entorhinal layer V neurons.

1068 Data are shown as mean \pm standard errors, and the statistical differences are shown in Figure 2 and Figure 2-figure supplement 2. 
A

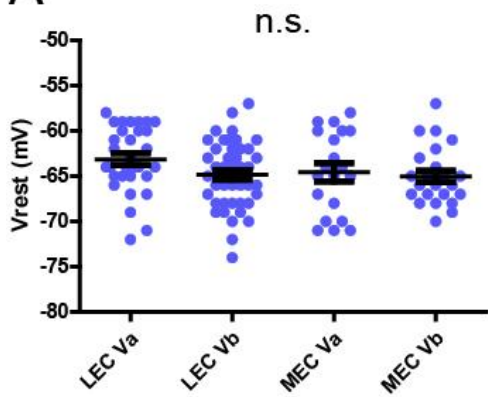

D

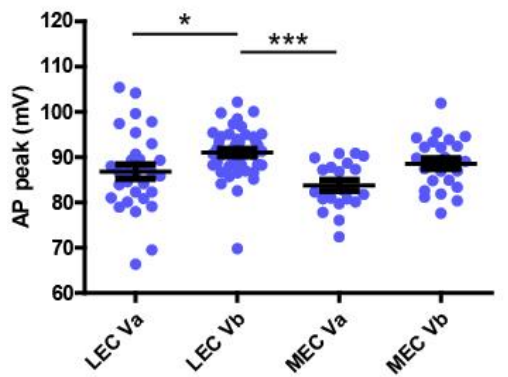

B

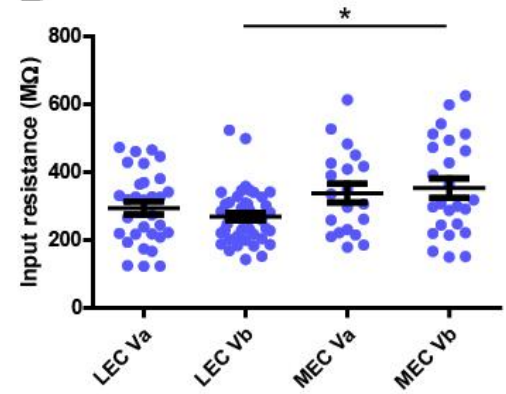

$E$

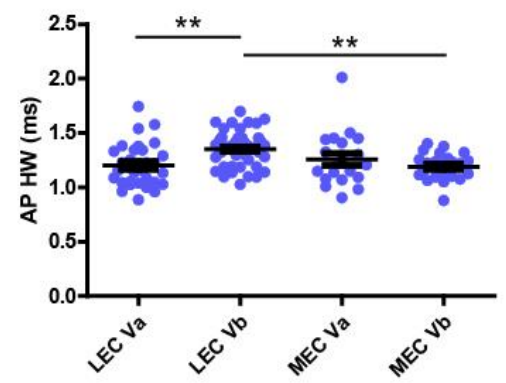

C

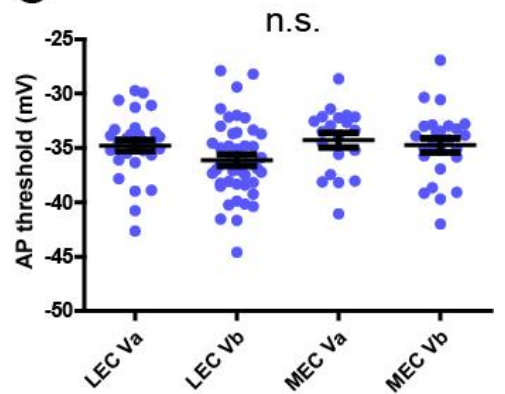

Figure 2-figure supplement 2. Electrophysiological features of $\mathrm{LVa} / \mathrm{LVb}$ neurons in

\section{LEC/MEC.}

1074 Differences of Vrest (A, one-way ANOVA, F3,117 $=1.694, \mathrm{p}=0.172)$, input resistance (B, one-way ANOVA, F3,117 $=4.006, * * p=0.0094$, Bonferroni's multiple comparison test, ${ }^{*} \mathrm{p}$ $<0.05)$, AP threshold $(\mathrm{C}$, one-way ANOVA, F3,117 = 2.116, $\mathrm{p}=0.102)$, AP peak (D, oneway ANOVA, F3,117 $=6.497, * * * \mathrm{p}=0.0004$, Bonferroni's multiple comparison test, $* \mathrm{p}<$ $0.05, * * * \mathrm{p}<0.001$ ), AP half width (E, one-way ANOVA, F3,117 $=6.099$, $* * * \mathrm{p}=0.0007$, Bonferroni's multiple comparison test, $\left.{ }^{* *} \mathrm{p}<0.01\right)$ between LEC-LVa $(\mathrm{N}=31)$, LEC-LVb $(\mathrm{N}=45)$, MEC-LVa $(\mathrm{N}=20)$, and MEC-LVb $(\mathrm{N}=25)$ neurons (Error bars: mean \pm standard errors). 
A
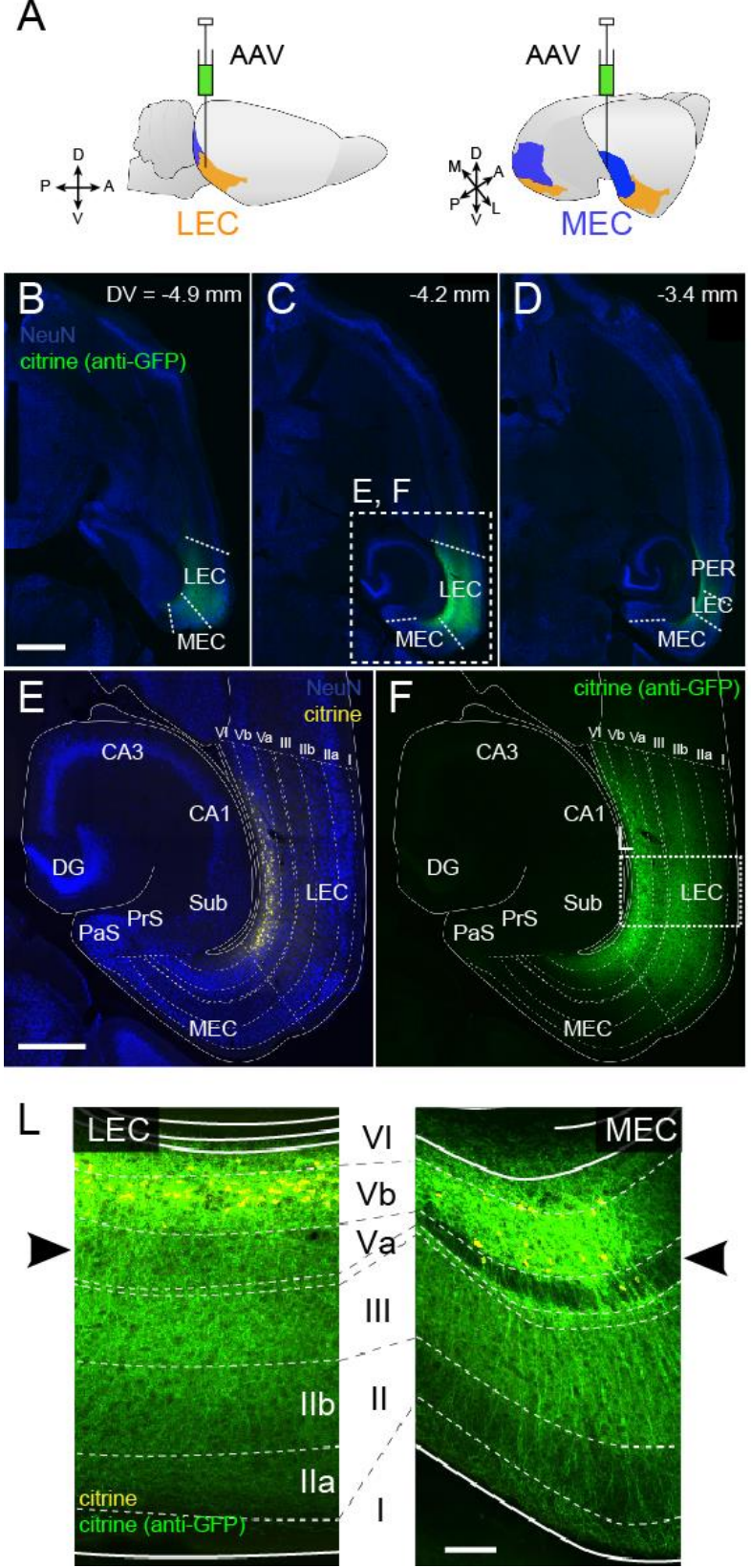

1) TG mice (MEC-13-53G)

- Enhancer - HSP68 MP - TTA -

2) tTA-dependent AAV

(AAV2/1-TRE-Tight-oChiEF-citrine)
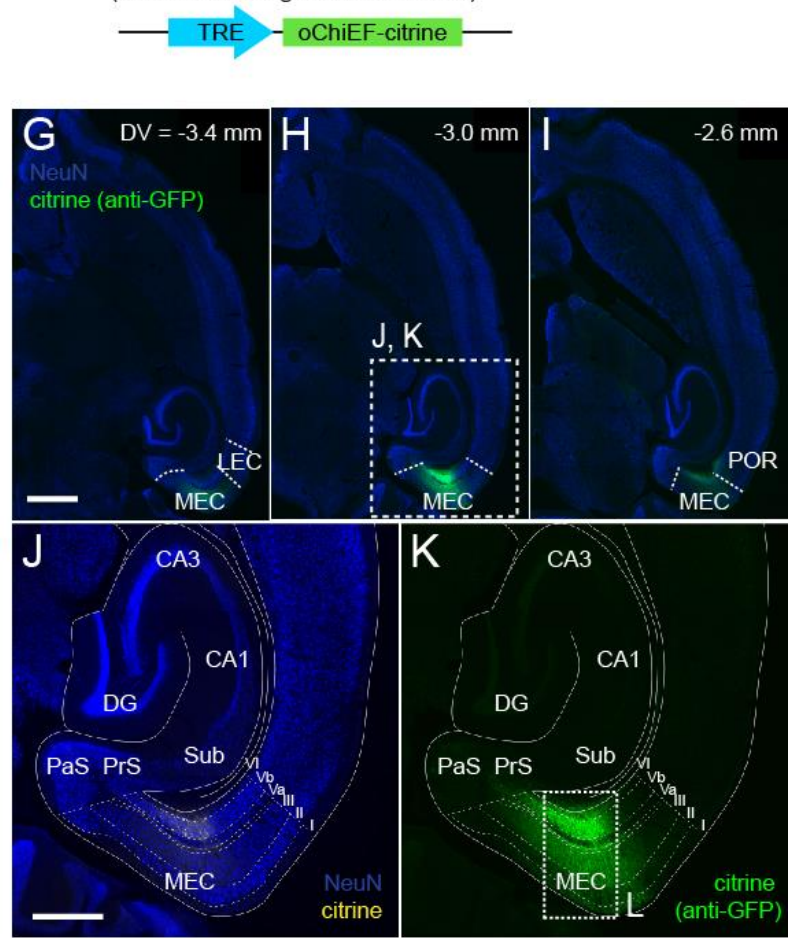

$\mathrm{M}$

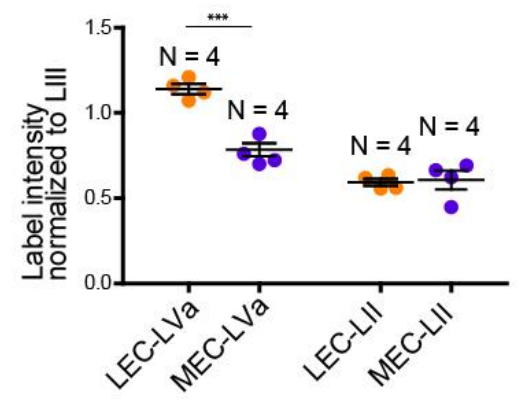

Figure 3. LVb neurons project locally, and their projections differ between LEC- and MEC.

1086 A. tTA-expressing entorhinal LVb neurons were visualized by injecting a tTA-dependent AAV expressing oChiEF-citrine into either LEC or MEC of MEC-13-53G. B-F, Horizontal sections showing distribution of labelled neurites originating from LEC-LVb at different dorsoventral levels (B-D). Images of EC (E, F) corresponds to the boxed area in (C). Note that the cell bodies of labelled neurons are located in LVb of LEC (citrine label in yellow, E), and that the labelled neurites mainly distribute within EC (citrine immunolabeling in green, F). The labelling observed in perirhinal cortex (PER; D) originate from the sparse infection of PER neurons due to the leakage of the virus along the injection tract. G-K, Horizontal sections showing distribution of labelled fibers originating from MEC-LVb at different dorsoventral level (G-I). Images of EC (J, K) corresponds to the boxed area in (H). Note that the cell bodies of labelled neurons are located in LVb of MEC (J), and that the labelled neurites mainly distribute within EC (K). The labelling observed in postrhinal cortex (POR; I) originate from the sparse infection of POR neurons due to the leakage of the virus along the 
injection tract. $\mathbf{L}$, Comparison of labelled neurites originating from LEC-LVb and MEC-LVb neurons (green), of which the cell bodies are visualized with citrine (yellow). The left panel corresponds to the boxed area in $(\mathrm{F})$ and is 90 degrees rotated to match the orientation of the right panel, which represent the boxed area in $(\mathrm{K})$. The distribution of the labelled fibers is strikingly different between LEC and MEC in LVa (black arrowhead) with a strong terminal projection in LEC and almost absent projections in LVa of MEC. M, Intensity of citrine immunolabeling in LVa and LII of LEC and MEC, normalized against the LIII labelling (Error bars: mean \pm standard errors, $\mathrm{N}=4$ ). The normalized labelling was significantly higher in LEC-LVa than in MEC-LVa (two-tailed paired t-test for LEC-LVa vs MEC-LVa: t6 = 7.68, $* * * p=0.0003$, LEC-LII vs MEC-LII: $\mathrm{t} 6=0.24, \mathrm{p}=0.82)$. Scale bars represent 1,000 $\mu \mathrm{m}$ for (B) and (G) (also apply to C, D, H, I), $500 \mu \mathrm{m}$ for (E) and (J) (also apply to F and K), $100 \mu \mathrm{m}$ for (L). Figure 3-source data 1. See also Figure 3-figure supplement 1. 

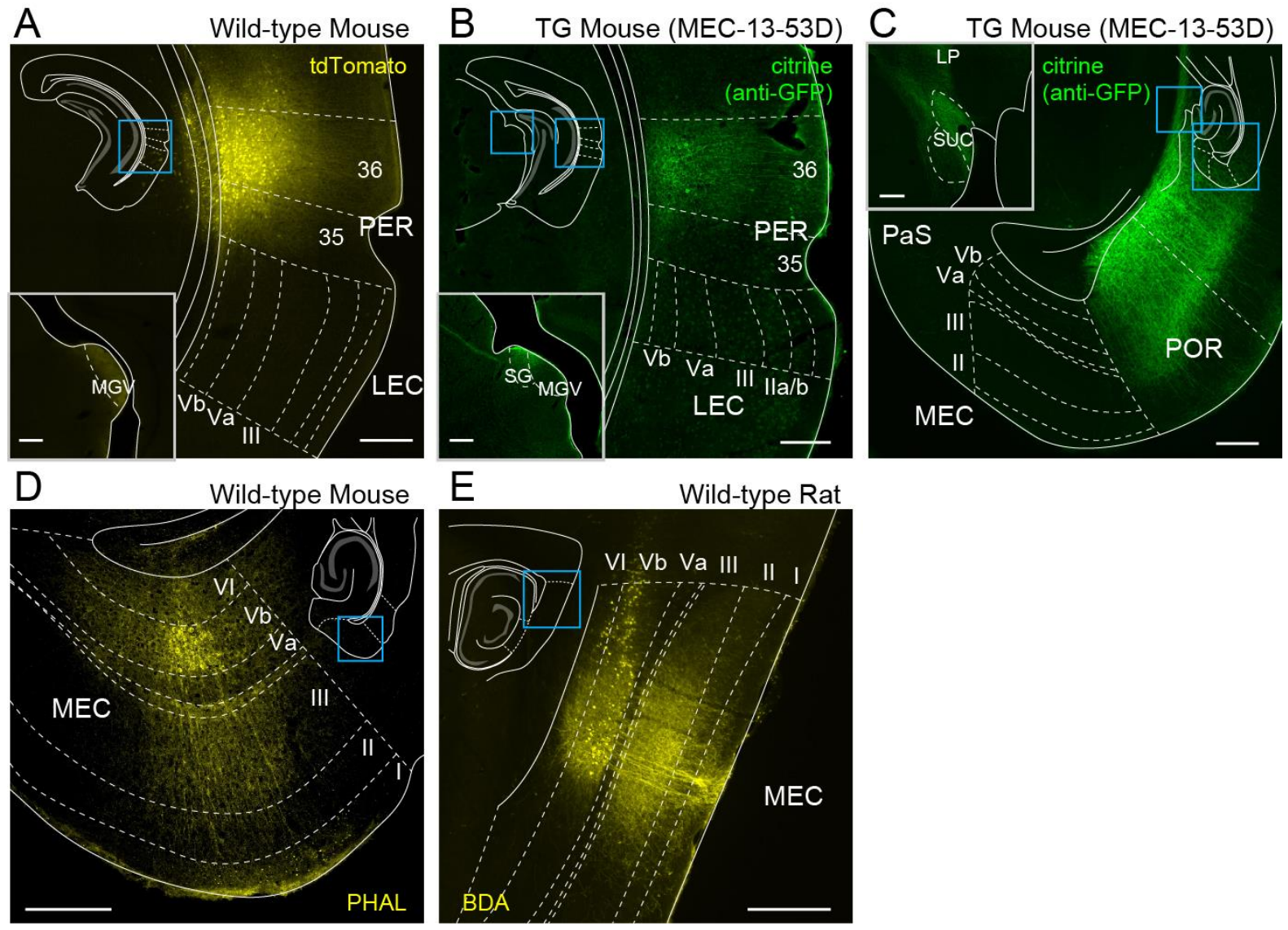

1112

Figure 3-figure supplement 1. Axonal distribution of PER, POR, and entorhinal LVb neurons.

A, Coronal section showing anterograde labelling following injection of AAV expressing tdTomato (AAV1.CAG.tdTomato.WPRE.SV40) into perirhinal cortex (PER) of wild-type mice. Note that labelled-axons are hardly observed in LEC, whereas dense labeling is observed in the thalamic ventral medial geniculate nucleus (MGV, inset). B, Coronal section showing anterograde labelling following injection of AAV expressing oChiEF-citrine (AAVTRE-tight-oChIEF-Citrine) into PER of MEC-13-53G. Similar to A, labeled-axons are hardly observed in LEC, whereas dense labeling observed in MGV and suprageniculate nucleus (SG) which are both known PER targets (inset). C, Horizontal section showing anterograde labelling following injection of AAV expressing oChiEF-citrine into postrhinal cortex (POR) of MEC-13-53G. Note that labeled-axons are hardly observed in MEC whereas dense labeling is observed in the lateralposterior nucleus (LP) and the superior colliculus (SUC) which are both known POR targets (inset). D, Horizontal section showing anterograde labelling of MEC-LVb neurons in wild-type mice. E, Sagittal section showing anterograde labelling of MEC-LVb neurons in wild-type rats. Note that, in both D and E, labelled axons are observed densely in LIII but sparsely in LVa. Schematics in the images give the location of each image. 35 , perirhinal area 35; 36, perirhinal area 36. 


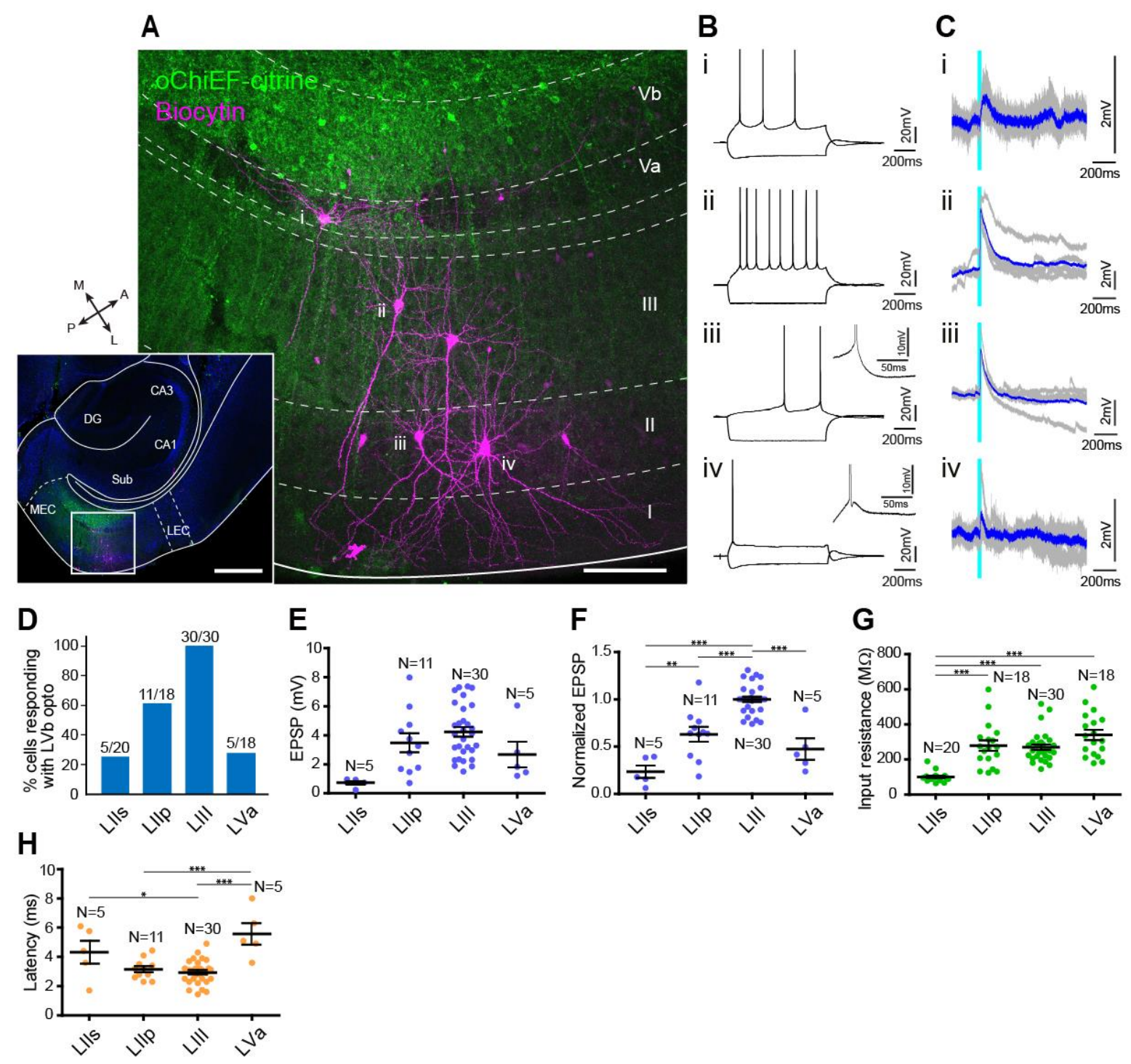

Figure 4. MEC-LVb neurons preferentially target LII/III pyramidal neurons.

A, Image of a representative horizontal slice showing expression of oChiEF-citrine in $\mathrm{LVb}$ neurons (green), and recorded neurons labelled with biocytin (magenta) in MEC. Inset shows a low power image of the section indicating the position of the higher power image. Scale bars represent $500 \mu \mathrm{m}$ (inset) and $100 \mu \mathrm{m}$. B, Voltage responses to injected current steps recorded from neurons shown in (A): i, pyramidal cell in LVa; ii, pyramidal cell in LIII; iii, pyramidal cell in LII; iv, stellate cell in LII. Inset in (iii) and (iv) shows the DAP in expanded voltage- and time-scale. Note that LII stellate cells (iv) show a clear sag potential and DAP compared to LII pyramidal cells (iii). C, Voltage responses to light stimulation (light blue line) recorded from neurons shown in (A). Average traces (blue) are superimposed on the individual traces (gray). D-G, The proportion of responding cells (D), EPSP amplitude (E), the normalized EPSP based on LIII response (F, one-way ANOVA, $F_{3,47}=33.29$, $* * * p<$ 0.0001 , Bonferroni's multiple comparison test, $* * p<0.01$, $* * * p<0.001$ ), and the input resistance $\left(\mathrm{G}\right.$, one-way ANOVA, $F_{3,82}=21.99$, *** $p<0.0001$, Bonferroni's multiple comparison test, $* * * p<0.001)$ were examined for each cell type (Error bars: mean \pm standard errors). H, Latency of EPSC onset for MEC neurons to optical activation (F, oneway ANOVA, $F_{3,47}=11.65, * * * p<0.0001$, Bonferroni's multiple comparison test, ${ }^{*} p<0.05$, 
source data 1. See also Figure 4-figure supplement 1, Figure 4-figure supplement 2, and Figure 4-figure supplement 3. 

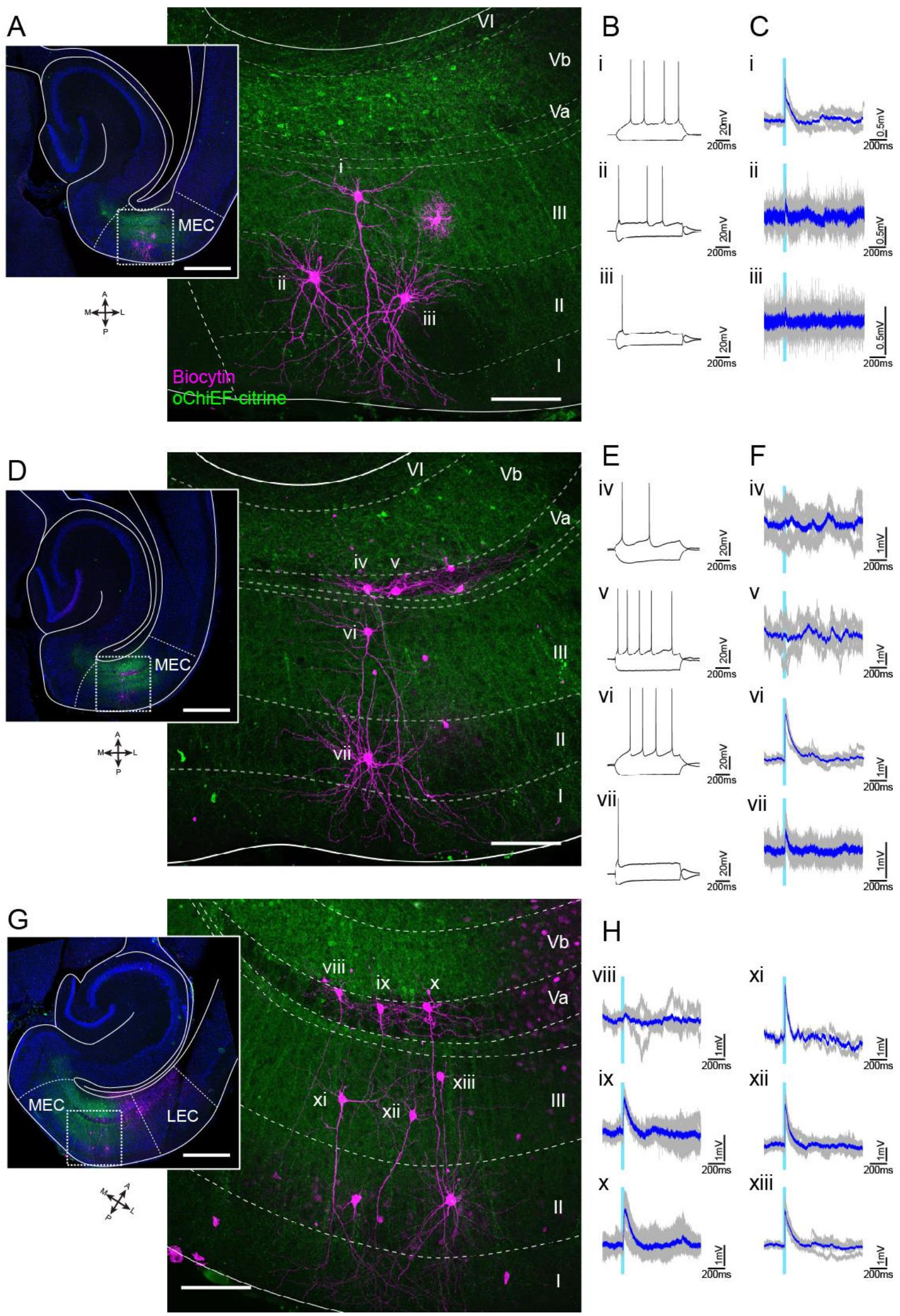

Figure 4-figure supplement 1. Representative patch-clamp recordings after optical stimulation of $\mathrm{LVb}$ fibers in MEC. 
1157 A-C, Horizontal MEC slice showing expression of oChiEF-citrine in LVb neurons (green), and biocytin-filled recorded layer II/III neurons (magenta, A). Voltage responses to injected current steps (B), and to light stimulation (light blue line, C) are shown for all three neurons (i, pyramidal neuron in LIII; ii,iii, stellate cells in LII). Average traces (blue) are superimposed on the individual traces (gray). D-F, Images of recorded MEC neurons (D) and their voltage responses (E, F; iv,v pyramidal cells in LVa; vi, pyramidal cell in LIII; vii, stellate cell in LII). G-H, Images of recorded MEC neurons (G) in LVa (Viii-x) and LIII (xixiii), and their voltage responses (H). This slice is from the animal shown in Figure 4, but it is more ventral than the one shown in Figure 4. Scale bars represent $500 \mu \mathrm{m}$ for insets, and 100 $\mu \mathrm{m}$ for higher power images. 


\section{A}
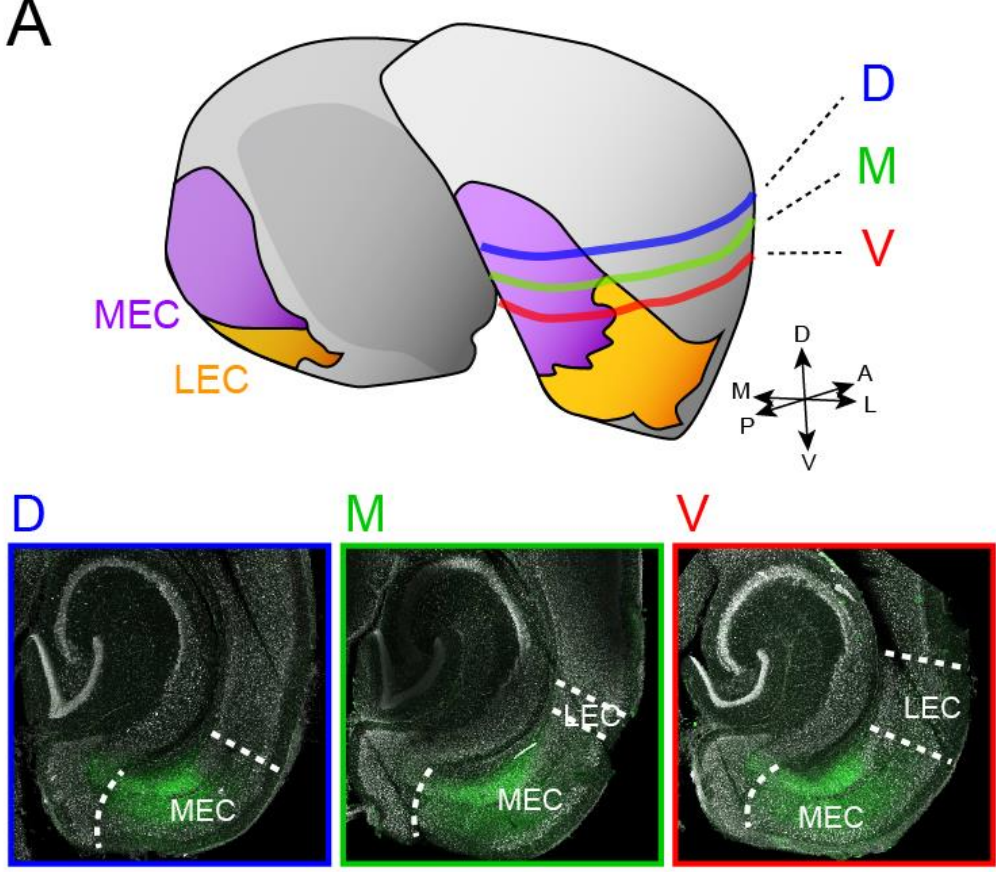
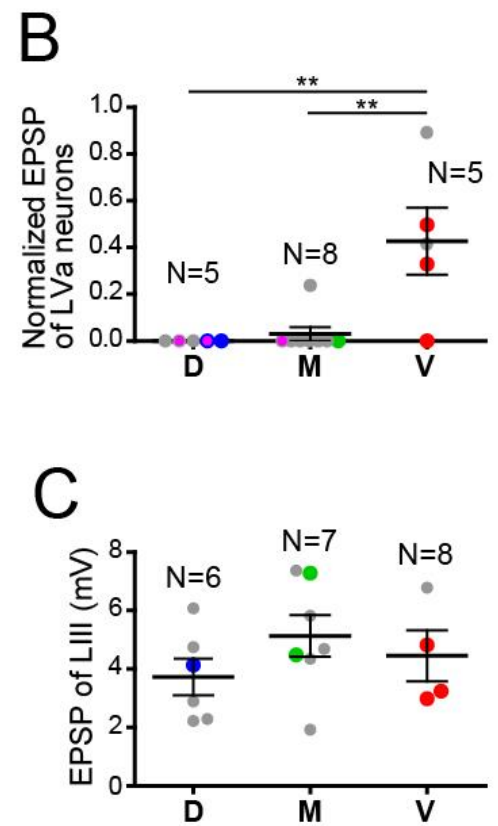

1168

1169

1170

1171

1172

1173

1174

1175

1176

1177

1178

1179

1180

1181

1182

1183

\section{Figure 4-figure supplement 2. Responses of MEC-LVa neurons at different} dorsoventral levels.

A, Schematic drawing and representative images showing three dorsoventral levels of slices in which the LVa neurons were recorded (D, dorsal level; M, medial level; V, ventral level). D (blue) corresponds to the sample shown in Figure 4-figure supplement 1 (D-F), while M (green) and V (red), which are taken from the same animal, correspond to the sample shown in Figure 4 and Figure 4-figure supplement $1(\mathrm{G}-\mathrm{H})$, respectively. B-C, Normalized EPSPs of LVa neurons (B) are significantly larger in more ventral slices (one-way ANOVA, F2,15 = 9.82, ${ }^{* * *} \mathrm{p}=0.0019$, Bonferroni's multiple comparison test, $\left.{ }^{* *} \mathrm{p}<0.01\right)$, whereas EPSPs of LIII neurons recorded at different dorsoventral level are indifferent $(\mathrm{C})$ (Error bars: mean \pm standard errors, one-way ANOVA, F2,14 = 1.045, $\mathrm{p}=0.3777$ ). Voltage responses recorded from the slice in (A) are shown in (B) and (C) as colored dots (blue dots in D, green dots in $\mathrm{M}$, and red dots in V). Magenta filled dots in (B) show LVa neurons with severed apical dendrites, indicating that severing the dendrites does not impact the absence of responses in dorsal MEC. Figure 4-figure supplement 2-source data 1. 
A
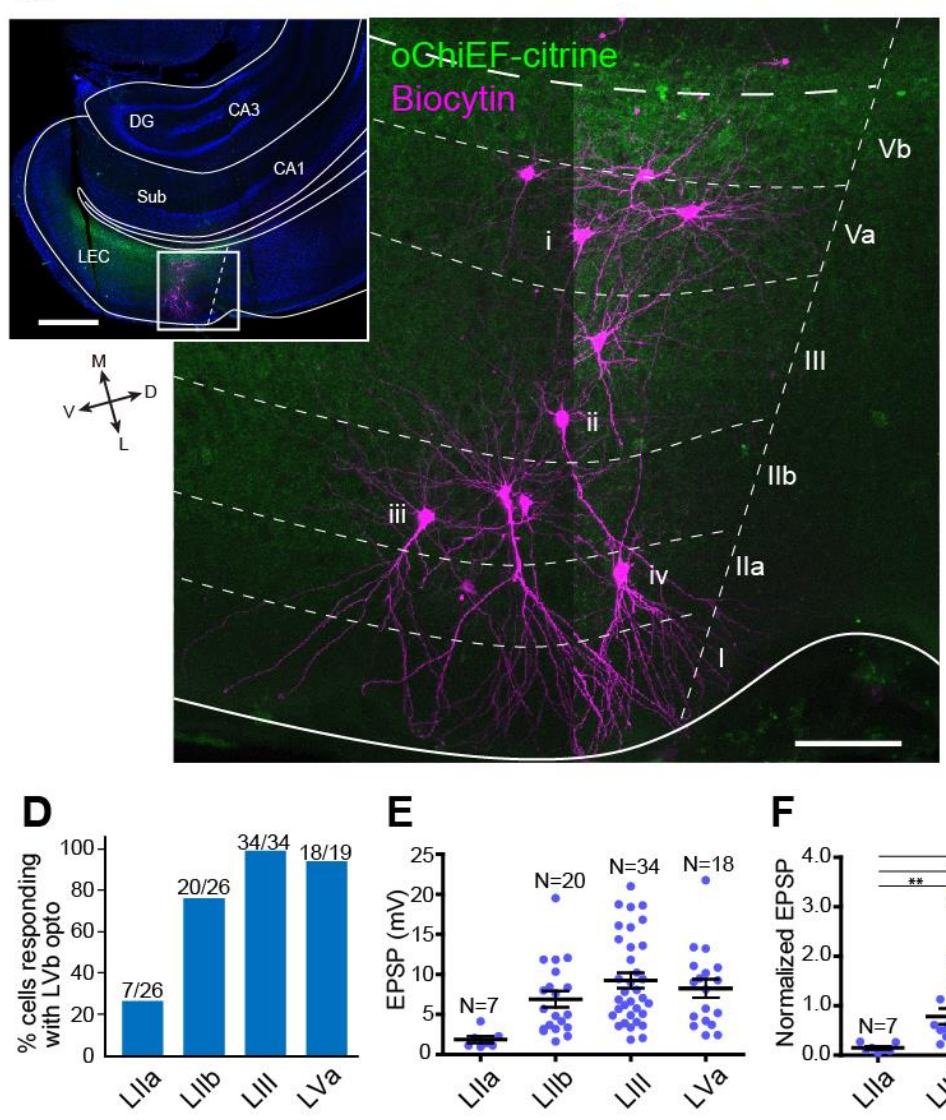

E

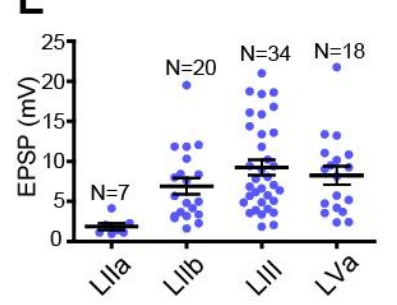

$\mathbf{F}$

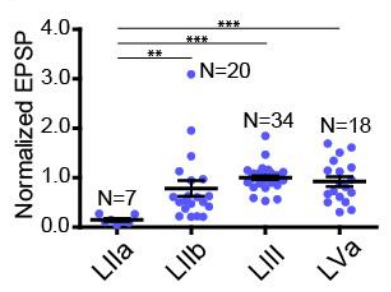

G

B
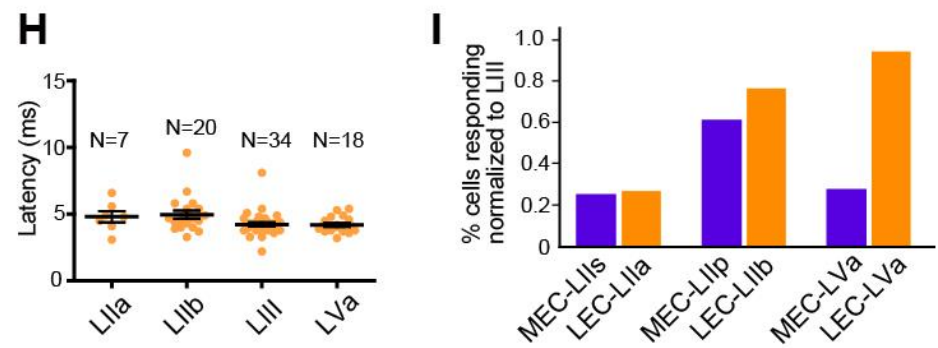

B

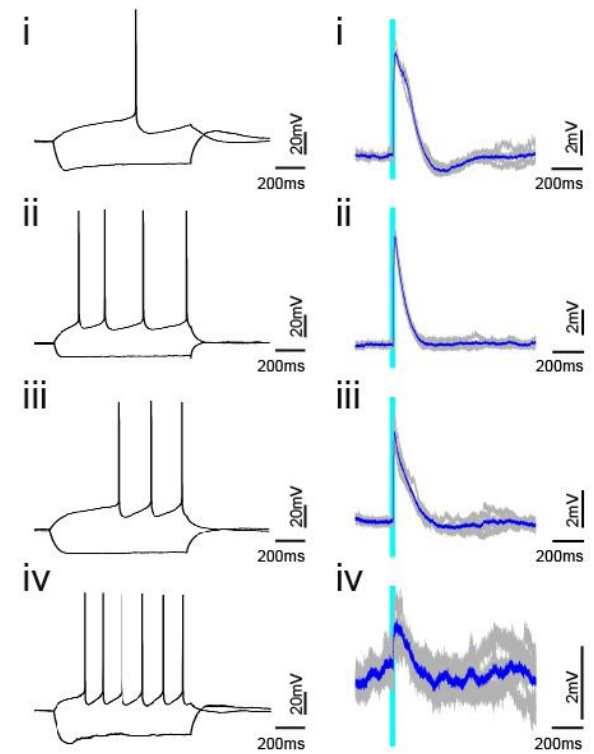

Figure 5. LEC-LVb neurons target LVa pyramidal neurons as well as LII/III pyramidal neurons.

A, Representative image of semicoronal slice showing expression of oChiEF-citrine in LVb neurons (green), and recorded neurons labelled with biocytin (magenta) in LEC. Inset shows a low power image of the section indicating the position of the higher power image. Scale bars represent $500 \mu \mathrm{m}$ (inset), and $100 \mu \mathrm{m}$. B, Voltage responses to injected current steps recorded from neurons shown in (A): i, pyramidal cell in LVa; ii, pyramidal cell in LIII; iii, pyramidal cell in LII; iv, fan cell in LII. C, Voltage responses to light stimulation (light blue line) recorded from neurons shown in (A). Average traces (blue) are superimposed on the individual traces (gray). D-G, The proportion of responding cells (D), EPSP amplitude (E), the normalized EPSP based on LIII response (F, one-way ANOVA, $F_{3,75}=7.675$, $* * * p=$ 0.0002 , Bonferroni's multiple comparison test, $* * p<0.01$, $* * * p<0.001$ ), and the input resistance $\left(\mathrm{G}\right.$, one-way ANOVA, $F_{3,101}=11.75$, $* * * p<0.0001$, Bonferroni's multiple comparison test, $* p<0.05, * * * p<0.001$ ) were examined for each cell type (Error bars: mean \pm standard errors). $\mathbf{H}$, Latency of EPSC onset for LEC neurons to optical activation (one-way 
normalized EPSP based on LIII response (J) between MEC and LEC (Error bars: mean \pm standard errors; two-tailed unpaired t-test, $\left.t_{21}=2.239,{ }^{*} p=0.0361\right)$. Figure 5-source data 1. See also Figure 5-figure supplement 1 and Figure 5-figure supplement 2. 

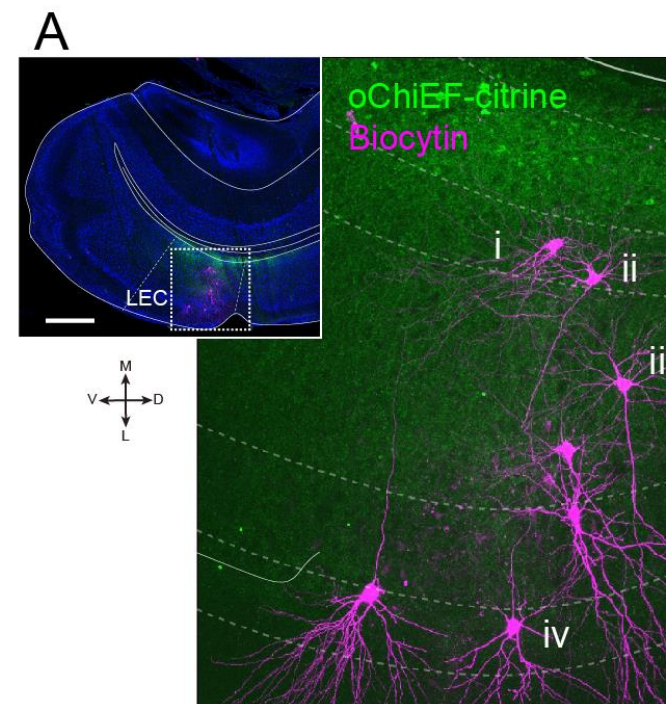

iv

Figure 5-figure supplement 1. Representative patch-clamp recording after optical stimulation of $\mathrm{LVb}$ fibers in LEC.

A, Semi-coronal LEC slice showing expression of oChiEF-citrine in LVb neurons (green), and biocytin-filled recorded layer II/III neurons (magenta). The inset indicates the position of the higher power image. Scale bars represent $500 \mu \mathrm{m}$ for inset and $100 \mu \mathrm{m}$ for high power image. B-C, Voltage responses to injected current steps (B), and to light stimulation (light blue line, C) recorded from neurons shown in (A) (i, ii, pyramidal cell in LVa; iii, pyramidal cell in LIII; iv, fan cell in LII). Average traces (blue) are superimposed on the individual traces (gray). 

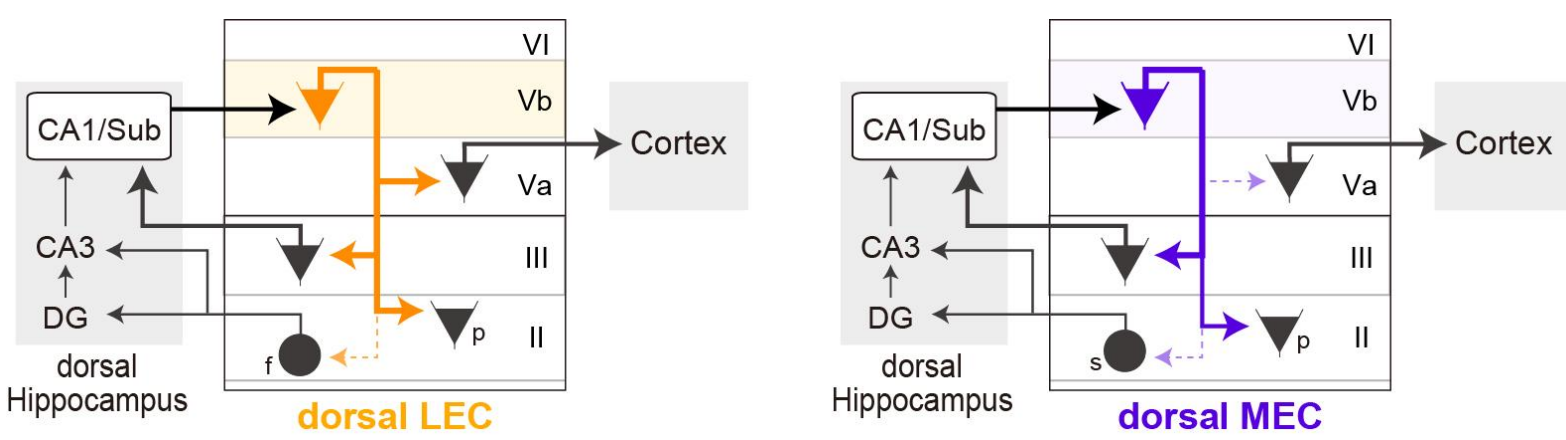

1218 Figure 6. Schematic diagram of the different local circuits in LEC and MEC used by 1219 LVb neurons to transfer dorsal hippocampal output.

Local connectivity of LVb neurons in LEC (left, orange) and MEC (right, purple). In both LEC and MEC, LVb neurons are the primary recipients of dorsal hippocampal output, but the transfer to LVa neurons through direct LVb-to-LVa projections is only prominent in LEC. Such projections are sparse and weak in MEC. Neurons in LVa are the output neurons of EC, projecting to the neocortex and other telencephalic subcortical structures. In contrast, in both LEC and MEC we find projections from LVb that target pyramidal cells in LIII, including neurons projecting to CA1 and subiculum, and pyramidal cells in LII. Projections to stellate (MEC) and fan (LEC) cells, which project to the dentate gyrus and CA3 are sparse and weak. The output projections of LII pyramidal neurons are not indicated in the figure, they project to ipsilateral-EC, contralateral-EC, CA1, or other telencephalic structures (Ohara et al., 2019). For clarity reasons all these projections are indicated schematically as originating from a single LVb neuron, but this is not yet known. Abbreviations: $f$, fan cell; s, stellate cell; $p$, pyramidal cell. 